Ann. Génét. Sél. anim., I973, 5 (I), 83-107.

\title{
ANALYSE STATISTIQUE DES PERFORMANCES D'ÉLEVAGE DES LAPINES. FACTEURS DU MILIEU, CORRÉLATIONS, RÉPÉTABILITÉS
}

\author{
R. ROUVIER, B. POUJARDIEU et J.-L. VRILLON \\ Laboratoive de Méthodologie génétique, \\ Centre de Recherches de Toulouse, I. N. R. A., \\ B. P. $n^{0} 12$ \\ 31320 Castanet-Tolosan
}

\section{RÉSUMÉ}

Une analyse statistique des performances d'élevage des lapines de race néo-zélandaise blanche et d'un élevage fauve de Bourgogne a été faite d'après les résultats de contrôle de performances des adhérents du Syndicat national d'Élevage et d'Amélioration du Lapin de Chair ( $594^{2}$ portées). Les caractères suivants ont été étudiés : nombre de lapereaux nés totaux et nés vivants par portée, taille de portée à $2 \mathrm{I}$ et 56 jours, taux de mortalité des lapereaux à la naissance, à $2 \mathrm{I}$ et 56 jours, poids total de portée à $2 \mathrm{I}$ et 56 jours, poids moyen individuel par portée des lapereaux à $2 \mathrm{I}$ et 56 jours, gain de poids du lapereau moyen de la portée entre $2 \mathrm{I}$ et 56 jours. Les effets des facteurs suivants, considérés comme fixes, et de leurs interactions, ont été testés ; année ( 4 années) et mois de naissance, numéro d'élevage, numéro d'ordre de la portée. Du fait de la présence d'interactions, nous avons dû nous ramener au test et à l'estimation des effets moisannée de naissance d'une part, numéro de portée (I à 5) d'autre part, pour chaque élevage séparément. L'étude des distributions de fréquence des données corrigées pour les effets de ces deux facteurs, pour chaque élevage séparément, a montré que les caractères relatifs à la prolificité et aux tailles de portée, notamment, s'écartent significativement de la normale et présentent une dissymétrie à gauche. Cela a une incidence sur le calcul de l'espérance du progrès génétique à la suite de la sélection.

Sur les mêmes données corrigées, une analyse hiérarchique de la variance a permis d'obtenir les composantes de la variance dues aux effets élevage, père et mère des portées.

L'élevage et la date de naissance des lapereaux modifient les performances zootechniques, ces deux facteurs étant en interaction. De mêrme, pour un élevage donné, il y a interactions entre les effets de l'année et du mois de mise bas. Les lapines ont leurs meilleurs résultats en portées numéros 3 et 4 . Les plus mauvais résultats sont obtenus chez les primipares chez lesquelles le taux de morts à la naissance, notamment, est très augmenté.

Les répétabilités dues aux effets des mères, pour les différents caractères, ont été calculées, de même que les corrélations. Les répétabilités mère sont significatives, quoique faibles, ce qui laisse supposər de faibles valeurs des héritabilités de ces caractères. Les effets de la mère agissent dans le même sens pour la viabilité et la croissance de leurs lapereaux jusqu'à l'âge de 56 jours. Un effet direct du père sur le nombre de lapereaux nés et nés vivants a été mis en évidence, ainsi que sur le taux de mortalité des lapereaux à la naissance.

Sur le plan phénotypique, la prolificité et les tailles de portée aux différents âges sont en corrélations négatives avec les poids moyens individuels par portée des lapereaux ( 21,56 jours). Cependant la vitesse de croissance du lapereau moyen de la portée, dans l'intervalle d'âge 2 I-56 jours, dépend peu de la prolificité et de la taille de portée. Une plus grande prolificité s'accompagne 
en moyenne, de tailles et poids de portée plus élevés à 2 I et 56 jours, une prolificité optimum se situant à IO-I 2 lapereaux nés totaux par portée. Les résultats obtenus ont permis de discuter l'intérêt des différents caractères mesurés dans le cadre du contrôle de performances, d'une part ; d'autre part, ils permettent de prévoir les méthodes de sélection sur les aptitudes maternelles applicables au lapin, bien qu'il reste encore à obtenir ou préciser les valeurs de leurs paramètres génétiques.

\section{IN'TRODUC'TION}

A la demande des éleveurs de lapins regroupés dans le Syndicat national d'Élevage et d'Amélioration du Lapin de Chair (S. N. E. A. L. C.) nous avons été amenés à définir en I962, en collaboration avec ce Syndicat et la Direction de l'Élevage du Ministère de l'Agriculture, un contrôle de performances, en ferme, du lapin de chair. Celui-ci porte sur les performances d'élevage des lapines et sur le poids individuel des lapereaux à l'âge de 56 jours. Les analyses des importances relatives des différents facteurs de variation (génétique et du milieu) ont permis, sur le plan pratique, d'établir pour les adhérents du S. N. E. A. L. C. une méthode de choix des meilleurs reproducteurs. Sa précision est limitée par le fait qu'il s'agit de données d'observations, les animaux à comparer ne l'étant pas dans des conditions comparables de milieu. Les résultats de ces analyses nous ont enfin permis d'établir les programmes rationnels de sélection du lapin de chair qui se mettent actuellement en place dans le cadre de stations de sélection. Les résultats sur les paramètres génétiques et du milieu pour des lapines soumises au contrôle de performances en ferme sont particulièrement originaux, contrairement à ce qui se passe pour d'autres espèces de mammifères domestiques étudiées depuis plus longtemps et dans un plus grand nombre de pays. Il nous a donc semblé utile d'analyser ces résultats de contrôle de performances du lapin. Dans cette étude, assez comparable à celle publiée par LEGAULT (I969) sur les performances d'élevage de truies de race Large-white, nous envisagerons l'influence des facteurs suivants sur les performances d'élevage des lapines :

- l'année et le mois de mise bas,

- l'élevage dans lequel la lapine a vécu,

- le numéro d'ordre de la portée,

- le père de la portée,

- la mère de la portée.

\section{MATÉRIEL ET MÉTHODE}

\section{I. - Origine et vérification des données}

Les performances d'élevage des lapines des adhérents du S. N. E. A. L. C. ont été soumises à partir de 1962 à un contrôle systématique effectué par les éleveurs eux-mêmes. La nécessité de répéter fréquemment la mesure des mêmes caractères au cours de la vie reproductive de la lapine, 
à des instants déterminés (pesées à âges fixes), le fait que la lapine est sensible à des changements fréquents de personnel la manipulant, interdisaient de confier ce travail à des techniciens circulant dans les élevages. Le point de départ du contrôle d'une portée est la déclaration de saillie (date de la saillie, numéros du mâle et de la femelle). Ensuite les résultats de la portée, lorsque la saillie est suivie de mise bas, sont enregistrés sur une fiche dont le modèle a été établi en fonction du traitement mécanographique routinier prévu. Au sevrage, les lapereaux sont identifiés individuellement à l'aide d'un numéro à 9 chiffres; les trois premiers chiffres correspondent au numéro de l'éleveur naisseur ; le quatrième chiffre représente le millésime de l'année de naissance ; les cinq chiffres suivants représentent un numéro d'ordre de naissance dans l'année, dans l'élevage.

Le technicien du Syndicat apporte son appui technique aux éleveurs pour la réalisation du contrôle de performances, s'assure de la validité des données fournies par l'éleveur (visite des troupeaux et vérification des identifications, effectifs, pesées...). Les fiches sont centralisées au Siège du Syndicat et envoyées à un atelier régional pour perforation. Enfin, un programme de calcul mécanographique, établi dès 1963 par les techniciens du centre de traitement de l'information du Département de Génétique animale au C. N. R. Z. à Jouy-en-Josas, vérifie la cohérence des données et notamment : durée de gestation, chronologie des dates enregistrées, effectifs de lapereaux par portée aux âges successifs, poids à âge-type, séquence des numéros de portées successives de chaque lapine.

\section{2. - Les variables étudiées}

Les variables suivantes relatives aux caractères de prolificité ou d'aptitudes maternelles ont été prises en considération. Elles correspondent, soit à des caractères directement mesurés, soit à des variables calculées. Elles concernent d'une part les caractères de prolificité et viabilité, d'autre part des caractères pondéraux :

$\mathrm{X}_{1}$ l'effectif total de lapereaux dans la portée à la naissance.

$\mathrm{X}_{2}$ le nombre de lapereaux nés vivants par portée.

$\mathrm{X}_{3}$ le nombre de lapereaux dans la portée à I'âge de $2 \mathrm{I}$ jours.

$\mathrm{X}_{4}$ le nombre de lapereaux dans la portée à l'âge de 56 jours.

$\mathrm{X}_{5}$ le taux de mortalité des lapereaux à la naissance.

$\mathrm{X}_{6}$ le taux de mortalité des lapereaux à $2 \mathrm{I}$ jours.

$X_{7}$ le taux de mortalité des lapereaux à 56 jours.

Les taux de mortalité sont calculés par portée et par rapport au nombre total de lapereaux nés.

$\mathrm{X}_{8}$ : le poids total de la portée à l'âge de $2 \mathrm{I}$ jours. La portée est pesée globalement à $2 \mathrm{r}$ jours \pm I jour, et le poids à $2 \mathrm{I}$ jours est calculé mécanographiquement en tenant compte d'une vitesse de croissance journalière moyenne des lapereaux et du nombre de lapereaux dans la portée.

$\mathrm{X}_{8}$ : le poids total de la portée à l'âge de 56 jours.

' Tous les lapereaux sont pesés individuellement à 56 jours \pm I jour et les poids sont ramenés au poids à l'âge de 56 jours, en tenant compte d'une vitesse de croissance journalière moyenne des lapereaux qui varie selon la race.

Des variables obtenues par le calcul à partir des caractères mesurés :

$\mathrm{X}_{10}$ : le poids moyen individuel par portée de lapereaux à l'âge de $2 \mathrm{r}$ jours (poids total de la portée divisé par le nombre de lapereaux dans la portée).

$\mathrm{X}_{11}$ : le poids moyen individuel par portée des lapereaux à l'âge de 56 jours (moyenne des poids individuels des lapereaux de la portée).

$\mathrm{X}_{12}$ : le gain de poids du lapereau moyen de la portée entre $2 \mathrm{I}$ et 56 jours.

\section{3. - Échantillonnage}

Les données présentement analysées ont été recueillies en ferme au cours des années I966, I967, I968 et 1969, dans 7 élevages. Nous avons choisi les élevages et années pour lesquels nous avions le plus de résultats avec une certaine continuité. Nous avons ainsi considéré les élevages pour lesquels nous avions au moins $r o 0$ portées contrôlées par an pendant 4 années successives.

Dans l'échantillon étudié nous avons des animaux de race Néo-Zélandaise blanche (6 élevages), Fauve de Bourgogne (I élevage) et un total de 5942 portées. Les portées de numéro supérieur à 5 (peu nombreuses et relatives à des lapines sélectionnées) ont été éliminées. Dans l'interprétation des données de croissance nous n'avons pris en considération que les portées pour lesquelles au moins un animal avait été contrôlé à l'âge de 56 jours. Pour les données de prolificité des femelles, effectifs par portée aux différents âges, viabilité des lapereaux, le seul critère d'élimination d'une portée était l'absence de lapereaux à la naissance. Le tableau I donne les valeurs moyennes par élevage pour l'ensemble des variables. 
R. ROUVIER, B. POUJARDIEU, J.-L. VRILLON

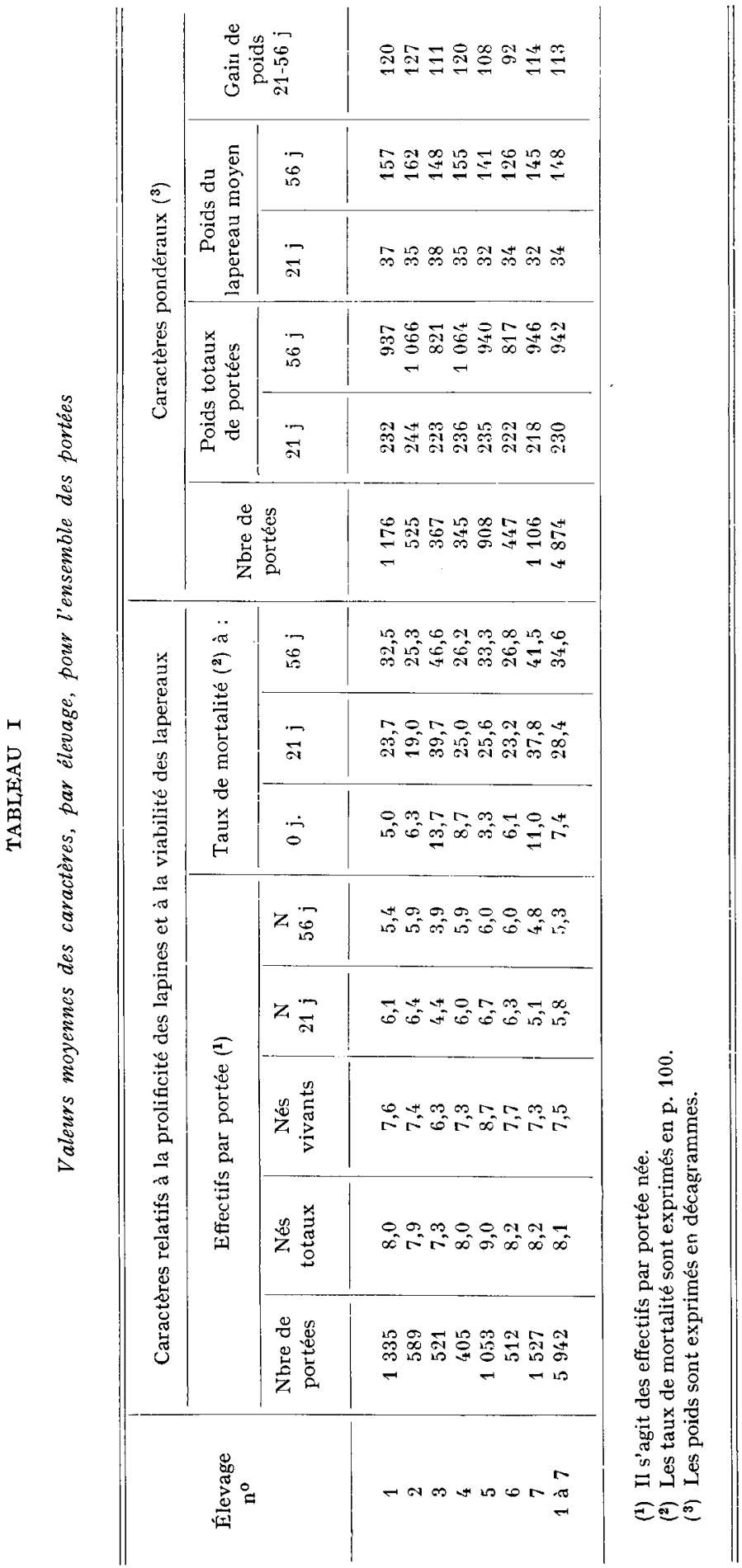




\section{4. - Modèle statistique d'analyse}

Dans une première série d'études nous avons effectué sur l'ordinateur IBM I620 du Département de Génétique animale, des analyses de variance à effets fixés, schéma croisé, modèle déséquilibré, pour tester la signification et l'interaction des effets suivants :

- l'année de mise bas ;

- le mois de mise bas ;

- l'élevage ;

- le numéro de portée de la lapine.

Les interactions étant significatives, cela interdisait l'interprétation de ces effets simples dans le cadre de ce modèle. Les résultats préliminaires de LEPLÈGE (communication personnelle) indiquaient l'existence d'un effet période de temps sur les caractères, pour des périodes aussi courtes que la durée d'un mois.

Nous avons donc été conduits à l'étude suivante :

Nous avons considéré chaque élevage séparément et utilisé intra élevage un modèle prenant en compte les effets fixés de la combinaison mois-année de mise bas ( 48 niveaux) et le numéro de portée (5 niveaux) :

$$
\mathrm{Y}_{i j k}=\mu+(\mathrm{AM})_{i}+(\mathrm{NP})_{j}+(\mathrm{AM}, \mathrm{NP})_{i j}+\mathrm{E}_{i j k}
$$

où $\mathrm{Y}_{i j k}$ représente la performance (chacune des variables $\mathrm{X}_{1}$ à $\mathrm{X}_{12}$ ) de la $k^{\text {ième }}$ lapine dans la catégorie $j$ ième mise bas $(j=\mathrm{I}$ à 5$)$ et $i$ ième mois-année $(i=\mathrm{I}$ pour le mois de janvier 1966 à 48 pour le mois de décembre 1969). (AM, NP) ${ }_{i j}$ est le terme d'interaction éventuelle entre les effets mois-année et numéros de portée. $\mu$ est une constante. Les $\mathrm{AM}_{l}(i=\mathrm{I}$ à 48$)$ sont les effets fixés du mois-année de mise bas et les $\mathrm{NP}_{j}(j=\mathrm{I}$ à 5$)$ sont les effets fixés du numéro de portée. $\mathrm{E}_{i j k}$ représente un résidu aléatoire indépendant des effets fixés considérés.

Le test de l'interaction ayant montré que celle-ci n'est pas significative, le modèle suivant a été utilisé :

$$
\mathrm{Y}_{i j k}=\mu+\mathrm{AM}_{i}+\mathrm{NP}_{j}+\mathrm{E}_{i j k}
$$

Le test des effets fixés ayant montré que ceux-ci étaient significatifs, ils ont été estimés, séparément pour chaque élevage, et on a calculé une nouvelle variable après correction pour ces effets.

$$
Z_{i j k}=\mathrm{Y}_{i j k}-\left(\widehat{\mathrm{AM}}_{i}+\widehat{\mathrm{NP}}_{\jmath}\right)
$$

Les $\widehat{\mathrm{AM}}_{i}$ et $\widehat{\mathrm{NP}}_{j}$ étant les estimations des effets fixés correspondants. Cette nouvelle variable, calculée pour chacun des caractères $\mathrm{X}_{1}$ à $\mathrm{X}_{12}$ et chaque élevage, n'est donc pas corrigée pour l'effet élevage. Nous avons analysé sa variance suivant le mođèle hiérarchique :

$$
\mathrm{Z}_{i j k l}=\mu+\mathrm{A}_{i}+\mathrm{B}_{i j}+\mathrm{C}_{i j k}+\mathrm{D}_{i j k l}
$$

dans le cas des résultats des animaux de race Néo-Zélandaise blanche, cette race étant représentée dans 6 des 7 élevages étudiés.

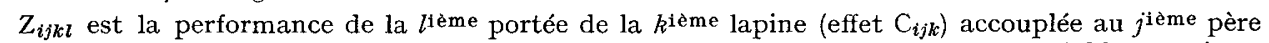
(effet $\mathrm{B}_{i j}$ ) dans l'élevage $i$ (effet $\mathrm{A}_{i}$ ). $\mathrm{D}_{i j k l}$ est un résidu aléatoire. Les diverses variables représentant les effets sont hiérarchisées les unes par rapport aux autres.

La race Fauve de Bourgogne étant observée dans un seul des 7 élevages, il n'est évidemment pas nécessaire d'introduire un effet élevage dans ce cas. Nous avons utilisé alors le modèle :

$$
Z_{j k l}=\mu+\mathrm{B}_{j}+\mathrm{C}_{j k}+\mathrm{R}_{j k l}
$$

$\mu$ est une constante, les effets $\mathrm{A}_{i}, \mathrm{~B}_{i j}, \mathrm{C}_{i j k}, \mathrm{D}_{i j k l}$ ou $\mathrm{B}_{j}, \mathrm{C}_{j k}, \mathrm{R}_{j k l}$ sont aléatoires et indépendants.

Ce modèle hiérarchique n'est valable que si des reproducteurs donnés sont utilisés dans un seul élevage et si chaque mère a donné toutes ses portées avec le même père. Cela représentait la majorité des cas. Il avait en effet été demandé aux éleveurs de réaliser ce plan d'accouplement. On aurait cependant pu analyser les données suivant un schéma hiérarchique et croisé, si nous avions disposé du programme de calcul adéquat. Nous avons donc dû éliminer pour cette analyse l'ensemble des performances de femelles servies par plus d'un mâle au cours de leur vie reproductive. Cette analyse hiérarchique nous a permis d'obtenir les composantes élevage $\left(\sigma_{A}^{2}\right)$, père $\left(\sigma_{\mathrm{B}}^{2}\right)$, mère $\left(\sigma_{\mathrm{C}}^{2}\right)$ et résiduelle $\left(\sigma_{\mathrm{D}}^{2}\right)$ de la variance. On a de même obtenu les composantes des covariances 
et les corrélations entre caractères dues à ces différents facteurs. On a pu calculer les répétabilités (corrélations entre les résultats de deux portées prises au hasard parmi toutes celles d'un père) dues aux effets père par le rapport:

$$
\frac{\sigma_{\mathbf{B}}^{2}}{\sigma_{\mathbf{B}}^{2}+\sigma_{\mathbf{C}}^{2}+\sigma_{\mathbf{D}}^{2}}
$$

et dues aux effets lapine intra père par le rapport :

$$
\frac{\sigma_{\mathrm{C}}^{2}}{\sigma_{\mathrm{C}}^{2}+\sigma_{\mathrm{D}}^{2}}
$$

Pour normaliser la distribution des taux de mortalité nous avons utilisé la transformation Arc sinus racine du pourcentage exprimant ce taux de mortalité. L'ensemble de ces calculs a fait appel à l'écriture d'un programme statistique par POUJARDIEU, permettant également l'enchaînement des opérations, sur l'ordinateur IBM $360 / 50$ du Centre de traitement de l'information au C. N. R. Z. à Jouy-en-Josas.

\section{RÉSULTATS}

\section{I. - Première série d'analyses}

Celle-ci porte sur les effets année de mise bas, mois de mise bas, numéro d'élevage, numéro de portée des lapines, considérés simultanément.

Les diverses analyses de variance ont montré l'existence d'interactions significatives entre certains de ces facteurs. Dans une année donnée, l'effet du mois de mise bas n'est pas le même pour tous les élevages, et dans un élevage donné l'effet du mois de mise bas n'est pas le même pour toutes les années.

\section{2. — Deuxième série d'analyses}

Une analyse de variance à deux facteurs de classification croisée, effectuée pour chacun des élevages séparément, a permis de mettre en évidence la signification des effets :

— année-mois de mise bas,

- numéro de portée.

L'interaction entre ces deux effets n'étant en général pas significative.

2. I. Tests des effets année-mois de mise bas et numéro de portée pour chaque élevage et leurs interactions.

Le tableau 2 donne les valeurs du $\mathrm{F}$ de Snedecor et sa signification pour le test des deux effets et de leur interaction, pour chaque élevage et les douze variables (tabl. 2).

La période de naissance des lapereaux (exprimée par la combinaison mois-année de naissance) a une influence le plus souvent significative sur les performances de croissance, les tailles de portée et les taux de mortalité aux différents âges. Cependant l'évolution temporelle des déviations liées aux niveaux de ce facteur diffère notablement d'un élevage à un autre pour un même caractère. Cela traduit l'existence d'inter- 


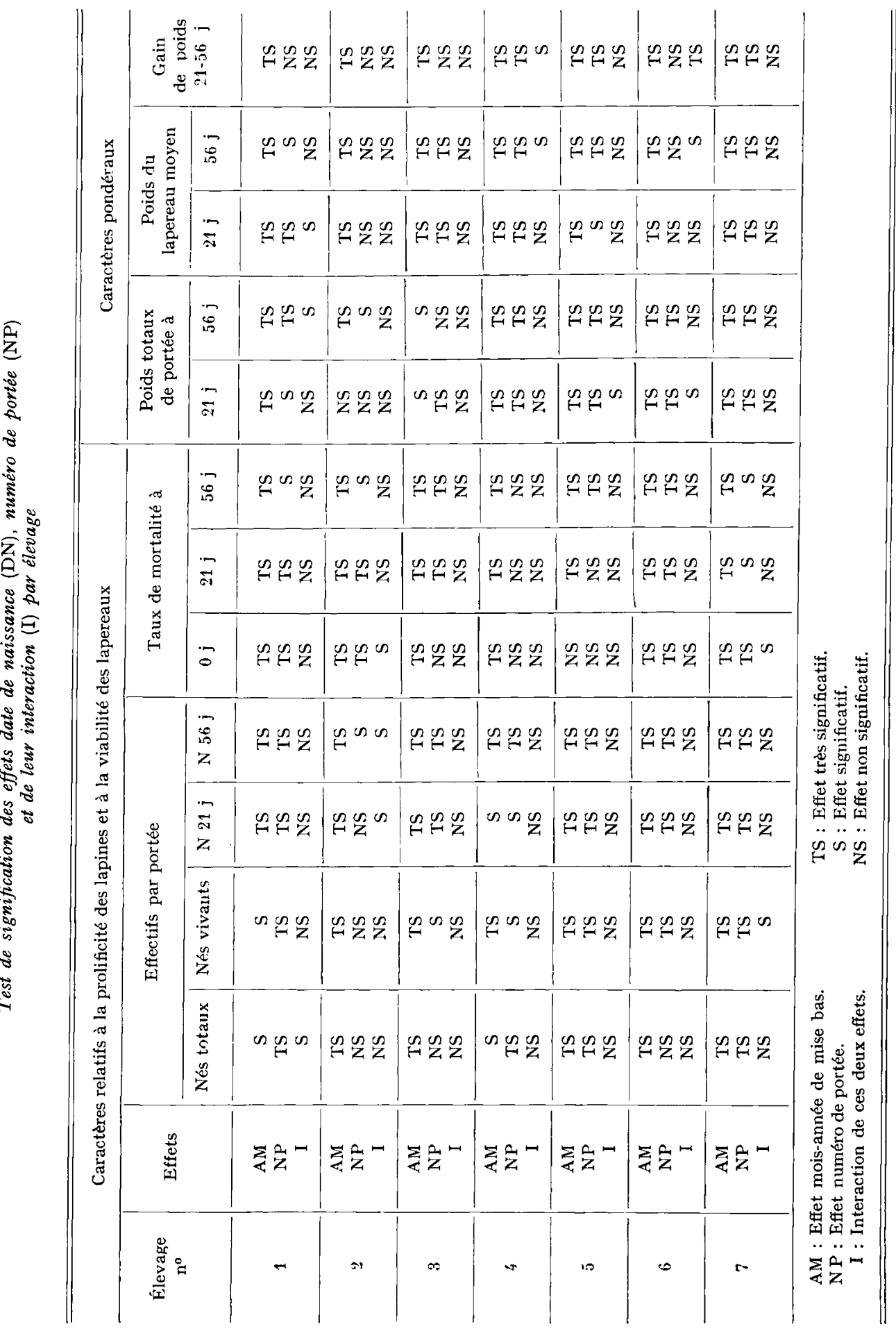


action entre ce facteur et l'effet de l'élevage. Il ne paraît pas nécessaire de donner les valeurs numériques des estimations des effets mois-année de naissance des 12 caractères pour les 7 élevages. L'évolution des performances en fonction de l'année, varie suivant les élevages. Cependant, en moyenne pour tous les élevages, la prolificité n'a pas augmenté de I966 à I969 (elle a même diminué en I969), mais les taux de mortalité à 2 I et 56 jours ont diminué, les poids totaux et moyens de portée à 2 I jours ont augmenté, ainsi que la taille de portée à 56 jours. Nous notons donc une tendance à l'amélioration des performances, qui se traduit par une meilleure viabilité des lapereaux, une augmentation de l'effectif par portée à l'âge de 56 jours (et corrélativement une légère diminution du poids des lapereaux à 56 jours), une amélioration de la production laitière des mères (tabl. 3).

Le numéro de portée de la lapine influe significativement, le plus souvent sur l'ensemble des performances analysées, sauf dans un élevage où cet effet n'est pas significatif sur un grand nombre de caractères. Les premières portées correspondent à des tailles de portée plus faibles et à des taux de mortalité plus élevés que pour les portées numéro 2 et plus. Les poids de portées sont les plus faibles en portée numéro I et les poids individuels sont inférieurs à la moyenne de l'élevage. Bien que l'évolution des effets numéro de portée soit parfois différente d'un élevage à l'autre, nous donnons dans le tableau 4 les valeurs moyennes des effets numéro de portée exprimées en pourcentage des valeurs moyennes des caractères, pour les 7 élevages.

\section{2. Effets pères et mères des portées.}

Le tableau 5 présente par race (Néo-Zélandais blanc et Fauve de Bourgogne) les valeurs des tests $\mathrm{F}$ de signification des effets des mères, les pourcentages de la variance attribuable à chacun des effets aléatoires et les répétabilités dues aux effets "mère " pour chacun des caractères. Ces résultats sont obtenus à partir des données préalablement corrigées pour les effets fixés année-mois de mise bas et numéro de portée.

Il existe des différences importantes entre élevages pour le poids individuel des lapereaux à 56 jours ( $25 \mathrm{p}$. Ioo de la variance) et le gain de poids de la portée de 2 I à 56 jours ( $27 \mathrm{p}$. IoO de la variance). Les différences entre élevages sont plus faibles pour les caractères de prolificité, taille de portée, viabilité des lapereaux, poids total de portée à l'âge de 2 I jours. L'effet du père est en général faible. Il faut cependant noter l'influence du père sur les nés vivants (II,7 p. Ioo de la variance) et le taux de mortalité à la naissance ( $5,30 \mathrm{p}$. Ioo de la variance) dans l'élevage Fauve de Bourgogne. Un effet sur les nés vivants de cet ordre de grandeur se retrouve dans 3 cas sur 6 lorsqu'on effectue une analyse par élevage en race Néo-Zélandaise blanche. On observe un effet père (faible) sur les poids moyens individuels des lapereaux aux âges de $2 \mathrm{I}$ et 56 jours, et sur le gain de poids du lapereau moyen de la portée entre $2 \mathrm{I}$ et 56 jours. Cet effet n'est pas mis en évidence en race Fauve de Bourgogne. L'effet de la mère est plus important que celui du père. La répétabilité liée à la mère varie, en race Néo-Zélandaise, de $\mathrm{I} 2 \mathrm{p}$. Ioo (poids total de la portée à 56 jours) à 20 p. Ioo (taux de mortalité à 56 jours). Elle est significative pour tous les caractères, à l'exception du taux de mortalité à la naissance. En race Fauve de Bourgogne, la répétabilité liée à la mère varie de ro $\mathrm{p}$. Ioo (taux de mortalité à 56 jours) à 38 p. Ioo (poids individuel moyen à 56 jours). Elle est significative pour tous les caractères, à l'exception des taux de mortalité à 21 et 56 jours. Ces répétabilités sont dans l'ensemble plus élevées qu'en race Néo-Zélandaise. 


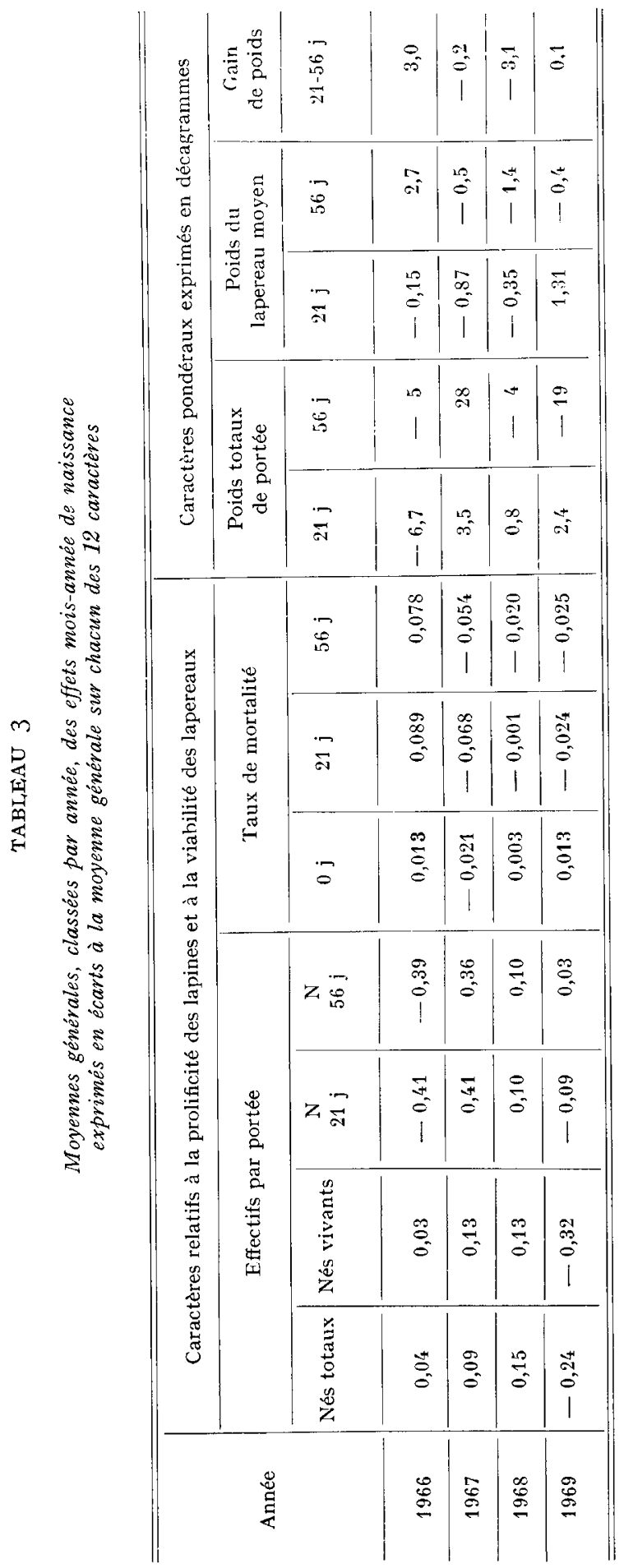




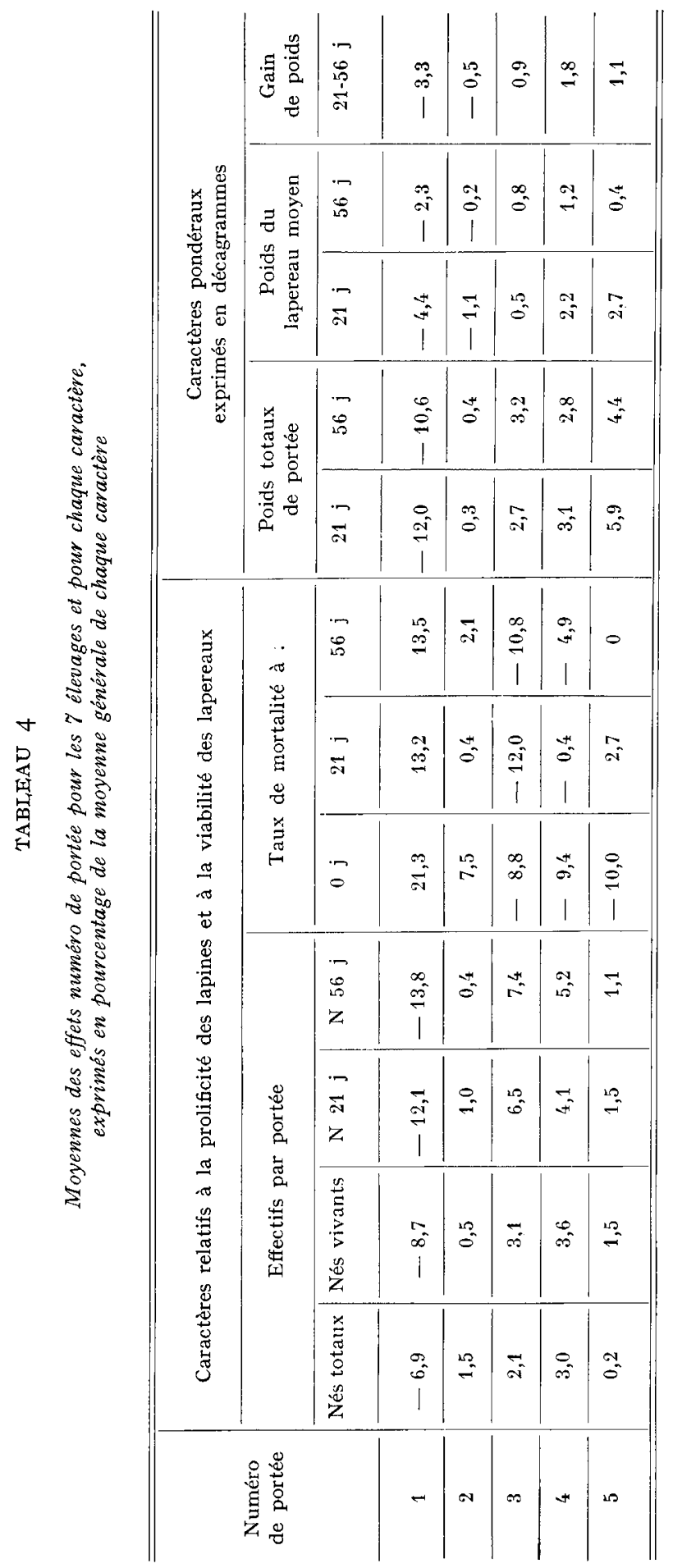




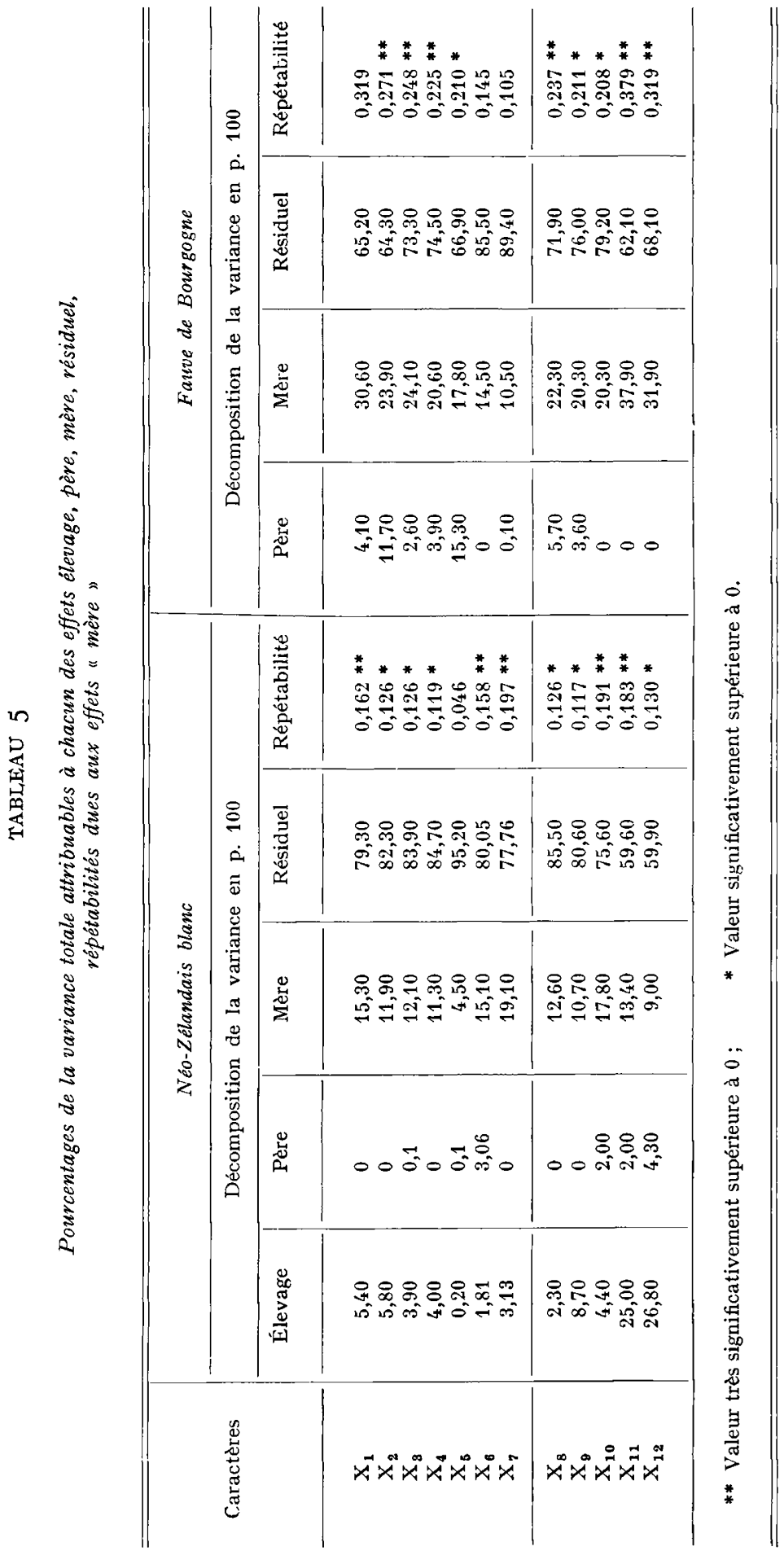




\section{3. Relations entre caractères.}

Les coefficients de corrélations linéaires phénotypiques et entre mères sont indiqués dans les tableaux 6 (Néo-Zélandais blanc) et 7 (Fauve de Bourgogne).

\section{31. Corrélations phénotypiques.}

Les performances de croissance (poids individuels moyens à 2 I et 56 jours, gain de poids du lapereau moyen de la portée entre $2 I$ et 56 jours) sont linéairement indépendantes des tailles de portée (Fauve de Bourgogne) ou en corrélation négative faible (Néo-Zélandais blanc). Par contre, les poids moyens individuels à 2 I et 56 jours sont en corrélation négative avec les tailles de portée aux différents âges, alors que les poids totaux de portée leur sont fortement corrélés positivement. Les portées lourdes à $2 \mathrm{I}$ jours le sont encore, en moyenne, à 56 jours $(r=0,72)$. Il y a une corrélation légèrement plus faible entre les poids moyen individuels à $2 \mathrm{I}$ et 56 jours. Les corrélations entre poids total et poids moyen individuel, à un âge donné, sont voisines de 0 .

Les effectifs des lapereaux aux différents âges sont linéairement liés. L’intensité de la liaison linéaire entre deux mesures successives de la taille de la portée passe par un minimum pour la combinaison des caractères, nombre de lapereaux nés vivants et taille de la portée à 2 I jours $(r=0,75$ en Néo-Zélandais et o,56 en Fauve de Bourgogne).

La taille des portées à la naissance est linéairement indépendante des taux mortalité à cet âge ( $r=0,03$ et 0,13 pour les deux races). Entre la naissance et 2I jours, la mortalité est plus importante dans les portées de forte taille à la naissance $(r=0,34$ et 0,26 avec les nés totaux), pour les deux races respectivement. Si l'on exclut le cas de la mortalité à la naissance, les taux de mortalité aux différents âges sont en corrélation négative avec les tailles de portée aux mêmes âges (2I et 56 jours). Les taux de mortalité aux âges successifs sont en corrélation positive, la plus forte corrélation étant entre les taux de mortalité à $2 \mathrm{I}$ et 56 jours $(r=0,83$ et 0,85$)$ pour les deux races.

\section{32. Corrélations entre caractères dues aux effets des mères.}

De même que l'on calcule une variance des effets des mères, on peut calculer une corrélation entre caractères due aux effets des mères. Ces effets sont génétiques et maternels, ainsi que dus au milieu commun aux animaux d'une même portée jusqu'au sevrage. Dans les deux races, tous les caractères pondéraux présentent des corrélations négatives avec les taux de mortalité à $2 \mathrm{I}$ et à 56 jours. Le taux de mortalité à la naissance se comporte différemment dans les deux races, dans ses relations avec les poids de portée. S'il est en corrélation négative avec les poids totaux de portée à 2 I et 56 jours chez les $N e ́ o-Z e ́ l a n d a i s ~ b l a n c s$, il ne présente aucune corrélation avec ces deux caractères dans la race Fauve de Bourgogne.

Les relations entre d'une part les poids totaux de portée à 2 I et 56 jours, la moyenne par portée des poids individuels des lapereaux à ces âges, d'autre part le gain de poids du lapereau moyen de la portée entre $2 I$ et 56 jours, sont différentes

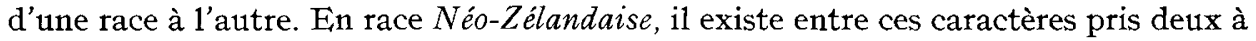
deux, des liaisons positives. En Fauve de Bourgogne, on observe entre poids totaux et poids individuels moyens de portée, la même absence de liaison linéaire qu'au niveau des corrélations phénotypiques. 


\begin{tabular}{|c|c|c|c|c|c|c|c|c|c|c|c|c|}
\hline$x^{ \pm}$ & 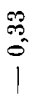 & $\begin{array}{l}\stackrel{0}{0} \\
0 \\
0 \\
1\end{array}$ & 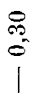 & $\begin{array}{l}\text { त् } \\
\text { o } \\
1\end{array}$ & $\begin{array}{l}\stackrel{o}{*}^{\circ} \\
0 \\
1\end{array}$ & $\stackrel{\ddot{\theta}}{\stackrel{0}{0}}$ & $\begin{array}{l}\frac{1}{6} \\
1\end{array}$ & $\begin{array}{l}\stackrel{\vec{N}}{\overrightarrow{0}} \\
\stackrel{0}{1}\end{array}$ & $\stackrel{5}{0}$ & 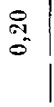 & $\begin{array}{l}\text { : } \\
\text { : }\end{array}$ & \\
\hline$\vec{x}$ & $\begin{array}{l}\vec{n} \\
\text { o } \\
\mid\end{array}$ & $\begin{array}{l}\text { Fi } \\
\text { ó } \\
i\end{array}$ & $\stackrel{\bar{s}}{\stackrel{50}{i}}$ & $\begin{array}{l}\stackrel{8}{*} \\
\dot{0} \\
i\end{array}$ & 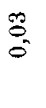 & $\stackrel{8}{\circ}$ & $\begin{array}{l}8 \\
0 \\
0 \\
1\end{array}$ & $\begin{array}{l}\overrightarrow{5} \\
\dot{0} \\
1\end{array}$ & $\vec{z}$ & $\begin{array}{l}\text { 点 } \\
\text { 足 }\end{array}$ & & $\begin{array}{l}\stackrel{20}{\circ} \\
0 \\
0\end{array}$ \\
\hline$\stackrel{0}{x^{2}}$ & $\begin{array}{l}\text { 总 } \\
0 \\
\text { | }\end{array}$ & $\begin{array}{l}\vec{b} \\
0 \\
1\end{array}$ & $\begin{array}{l}\dot{0} \\
\theta_{0} \\
1\end{array}$ & 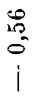 & $\stackrel{\varphi}{0}$ & $\stackrel{8}{\circ}$ & $\bar{g}$ & $\frac{12}{0}$ & 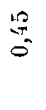 & & $\begin{array}{l}18 \\
0 \\
0 \\
0\end{array}$ & $\begin{array}{l}18 \\
0 \\
0\end{array}$ \\
\hline$\ddot{x}$ & $\begin{array}{l}\text { în } \\
\text { : }\end{array}$ & $\begin{array}{l}20 \\
200 \\
0 \\
0\end{array}$ & 芯 & $\begin{array}{l}\infty \\
\infty \\
0 \\
0\end{array}$ & $\stackrel{\vec{m}}{\dot{m}}$ & $\begin{array}{l}\ddot{i} \\
\dot{i} \\
\dot{0}\end{array}$ & 总 & $\stackrel{N}{0}$ & & 苚 & : & $\tilde{\sigma}$ \\
\hline$x^{\infty}$ & $\stackrel{\infty}{\stackrel{\infty}{0}}$ & $\underset{+}{\stackrel{+}{+}}$ & $\stackrel{2}{2}$ & $\begin{array}{l}\infty \\
: \\
0\end{array}$ & 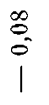 & $\begin{array}{l}\stackrel{2}{0} \\
0 \\
0 \\
1\end{array}$ & $\begin{array}{l}0 \overline{0} \\
0 \\
0 \\
1\end{array}$ & & $\stackrel{i n}{0}$ & $\begin{array}{l}\mathbb{N} \\
\stackrel{0}{=}\end{array}$ & $\stackrel{\infty}{\stackrel{\infty}{\sigma}}$ & 苟 \\
\hline$\tilde{x}$ & âd & $\frac{\pi}{0}$ & $\begin{array}{l}\text { a } \\
\text { o } \\
1\end{array}$ & $\begin{array}{l}\infty \\
\stackrel{\infty}{0} \\
\stackrel{0}{1} \\
1\end{array}$ & 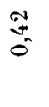 & $\begin{array}{l}\stackrel{\infty}{\infty} \\
0 \\
0\end{array}$ & & 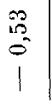 & $\begin{array}{l}\overrightarrow{\sigma^{*}} \\
\dot{\theta}^{\prime} \\
1\end{array}$ & $\begin{array}{l}0 \\
0 \\
0 \\
0 \\
0\end{array}$ & $\begin{array}{l}\infty \\
\tilde{\sigma}_{0}^{\prime} \\
0\end{array}$ & 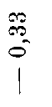 \\
\hline$\dot{x}^{0}$ & 苟 & $\stackrel{\infty}{\stackrel{0}{0}}$ & $\begin{array}{l}\infty \\
\dot{m} \\
\stackrel{\infty}{\infty}\end{array}$ & 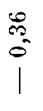 & $\stackrel{1}{0}$ & & $\frac{1}{5}$ & $\begin{array}{l}\bar{\infty} \\
\hat{\theta}^{\prime} \\
1\end{array}$ & $\begin{array}{l}0 \\
\infty \\
0 \\
0 \\
0\end{array}$ & $\begin{array}{l}8 \\
0\end{array}$ & $\stackrel{\infty}{\stackrel{\infty}{0}}$ & $\begin{array}{l}\stackrel{8}{\text { N }} \\
\stackrel{0}{1} \\
1\end{array}$ \\
\hline$x^{n}$ & $\stackrel{\dddot{g}}{\circ}$ & $\begin{array}{l}\text { If } \\
\text { : }\end{array}$ & $\begin{array}{l}\text { ज्ञ } \\
\text { o } \\
\text { | }\end{array}$ & $\begin{array}{l}\text { ap } \\
0 \\
0 \\
0 \\
1\end{array}$ & & $\begin{array}{l}8 \\
8 \\
0 \\
1\end{array}$ & $\begin{array}{l}\text { ôn } \\
\text { ô } \\
1\end{array}$ & $\begin{array}{l}8 \\
\dot{\theta} \\
0 \\
1\end{array}$ & $\begin{array}{l}\infty \\
\stackrel{0}{0} \\
0 \\
1\end{array}$ & $\tilde{\sigma}$ & $\stackrel{\infty}{0}$ & $\frac{12}{0}$ \\
\hline$x$ & $\begin{array}{c}\text { in } \\
\text { ing } \\
0\end{array}$ & $\begin{array}{l}18 \\
6 \\
0\end{array}$ & $\begin{array}{l}\infty \\
\infty \\
0\end{array}$ & & $\stackrel{8}{0}$ & $\begin{array}{l}\text { in } \\
\text { o } \\
\text { i }\end{array}$ & $\begin{array}{l}80 \\
0 \\
0 \\
0\end{array}$ & $\begin{array}{l}10 \\
0 \\
0 \\
0\end{array}$ & $\stackrel{\vec{s}}{\rightarrow}$ & $\begin{array}{l}8 \\
0 \\
0\end{array}$ & $\bar{\pi}$ & $\begin{array}{l}\text { 잉 } \\
\text { on } \\
0\end{array}$ \\
\hline$\ddot{x}^{*}$ & $\begin{array}{l}\dot{B} \\
\dot{0}\end{array}$ & $\frac{12}{2}$ & & 跑 & $\stackrel{\infty}{0}$ & $\begin{array}{l}\text { so } \\
\text { is } \\
\text { o } \\
1\end{array}$ & 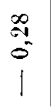 & $\begin{array}{l}\stackrel{8}{0} \\
0\end{array}$ & $\underset{\dddot{N}}{\stackrel{N}{0}}$ & $\begin{array}{l}\bar{a} \\
0 \\
0 \\
1\end{array}$ & $\stackrel{\infty}{\overrightarrow{0}}$ & $\frac{O}{0}$ \\
\hline$\ddot{x}$ & $\begin{array}{l}\ddot{2} \\
\dot{\theta}\end{array}$ & & $\underset{+}{\stackrel{9}{*}}$ & $\begin{array}{l}\mathscr{8} \\
i \\
1\end{array}$ & $\stackrel{2}{0}$ & $\vec{E}$ & $\begin{array}{l}\mathbb{\infty}_{\infty} \\
0\end{array}$ & $\stackrel{\circ}{\circ}$ & $\underset{\mid}{\stackrel{\theta}{e}}$ & 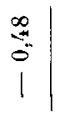 & $\begin{array}{l}0 \\
o 1 \\
0 \\
1\end{array}$ & $\frac{0}{0}$ \\
\hline$x^{-1}$ & & $\stackrel{\circ}{=}$ & 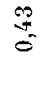 & $c_{0}^{+t}$ & \begin{tabular}{l}
\multirow{N}{*}{} \\
$\stackrel{0}{0}$
\end{tabular} & 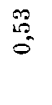 & $\begin{array}{l}\stackrel{N}{5} \\
0\end{array}$ & $\stackrel{20}{0}$ & $\begin{array}{l}\stackrel{5}{0} \\
1\end{array}$ & $\begin{array}{l}\stackrel{\leftrightarrow}{*} \\
\stackrel{\leftrightarrow}{0} \\
1\end{array}$ & $\begin{array}{l}\text { â } \\
\text { a } \\
0 \\
1\end{array}$ & $\begin{array}{l}8 \\
\dot{\theta} \\
0_{0}\end{array}$ \\
\hline & $\vec{x}$ & $x^{*}$ & $x^{\infty}$ & $\vec{x}$ & $x^{n}$ & $x^{0}$ & $\dot{x}$ & $x^{\infty}$ & $\ddot{x}$ & $x^{\circ}$ & $\bar{x}$ & $\stackrel{A}{\vec{x}}$ \\
\hline 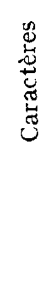 & 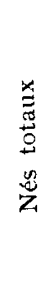 & 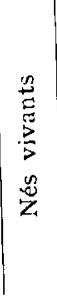 & $\begin{array}{l}m \\
z\end{array}$ & $\begin{array}{l}m \\
15 \\
z\end{array}$ & $\stackrel{\vdots}{0}$ & 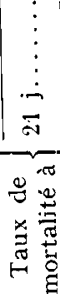 & $\stackrel{\dot{0}}{\stackrel{\circ}{\text { in }}}$ & 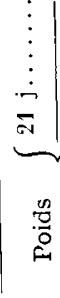 & 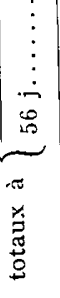 & $\left|\begin{array}{c}\vdots \\
\frac{\vdots}{\sigma 1}\end{array}\right|$ & 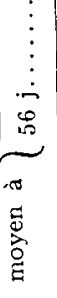 & 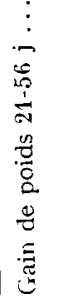 \\
\hline
\end{tabular}




\begin{tabular}{|c|c|c|c|c|c|c|c|c|c|c|c|c|}
\hline$\ddot{x^{2}}$ & $\begin{array}{l}0 \\
0 \\
0 \\
1\end{array}$ & $\begin{array}{l}\hat{0} \\
\dot{0} \\
\dot{0}\end{array}$ & 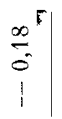 & $\begin{array}{l}0 \\
0 \\
0\end{array}$ & $\begin{array}{l}E \\
\vdots \\
1\end{array}$ & $\bar{z}$ & $\begin{array}{l}0 \\
0 \\
0 \\
1\end{array}$ & $\stackrel{e}{\circ}$ & $\stackrel{\overbrace{}}{\stackrel{0}{0}}$ & 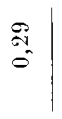 & $\begin{array}{l}0 \\
\infty \\
0 \\
0\end{array}$ & \\
\hline$\vec{x}$ & 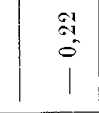 & 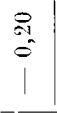 & 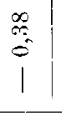 & $\stackrel{\stackrel{s}{0 !}}{=}$ & $\begin{array}{l}8 \\
8 \\
1\end{array}$ & $\stackrel{\infty}{=}$ & $\hat{\tilde{\theta}}$ & $\stackrel{e}{0}$ & $\mathscr{O}_{0}^{0}$ & $\begin{array}{l}\dot{\theta} \\
\dot{0}\end{array}$ & & $\vec{E}$ \\
\hline$\stackrel{0}{\vec{x}}$ & $\stackrel{2}{2}$ & \begin{tabular}{l}
0 \\
\multirow{2}{*}{} \\
0 \\
1
\end{tabular} & $\begin{array}{l}\text { a } \\
\text { ind } \\
0 \\
0 \\
1\end{array}$ & $\stackrel{8}{\stackrel{+}{\circ}}$ & $\stackrel{5}{0}$ & 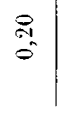 & $\begin{array}{l}x \\
0 \\
0 \\
0\end{array}$ & $\frac{\theta}{0}$ & 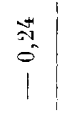 & & $\stackrel{\Xi}{=}$ & $\stackrel{\infty}{=}$ \\
\hline$x^{\infty}$ & $\begin{array}{l}\infty \\
\stackrel{0}{0} \\
0\end{array}$ & $\stackrel{\infty}{\stackrel{\infty}{0}}$ & 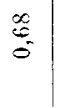 & $\begin{array}{l}\infty \\
\infty \\
0\end{array}$ & $\stackrel{\Delta}{0}$ & 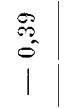 & $\begin{array}{l}8 \\
8 \\
0 \\
1\end{array}$ & $\stackrel{i}{\stackrel{0}{\circ}}$ & & $\stackrel{8}{0}$ & $\stackrel{8}{0}$ & $\stackrel{8}{g}$ \\
\hline ङूँ & 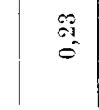 & 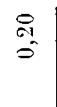 & $\begin{array}{l}8 \\
0 \\
0\end{array}$ & $\begin{array}{l}8 \\
0 \\
0\end{array}$ & $\begin{array}{l}5 \\
8 \\
0\end{array}$ & $\begin{array}{l}0 \\
0 \\
0 \\
1\end{array}$ & $\begin{array}{l}5 \\
0 \\
1\end{array}$ & & $\stackrel{5}{\circ}$ & $\frac{0}{0}$ & $\begin{array}{l}\infty \\
0 \\
0\end{array}$ & $\begin{array}{l}\vec{\sigma} \\
0 \\
0\end{array}$ \\
\hline 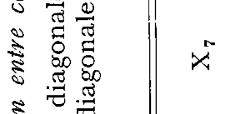 & $\begin{array}{l}\stackrel{\Delta}{*} \\
\stackrel{0}{0}\end{array}$ & $\frac{m}{0}$ & $\begin{array}{l}\overline{3} \\
\stackrel{8}{8} \\
1\end{array}$ & $\stackrel{R}{i}$ & $\stackrel{\stackrel{8}{1}}{\stackrel{0}{0}}$ & $\begin{array}{l}\infty \\
\stackrel{\infty}{\infty} \\
0\end{array}$ & & $\stackrel{\infty}{0}$ & $\begin{array}{c}\substack{\infty \\
10 \\
0 \\
0 \\
1} \\
\end{array}$ & $\begin{array}{l}\text { ज̃ } \\
\stackrel{0}{1} \\
1\end{array}$ & $\stackrel{\infty}{\infty}$ & $\begin{array}{l}\text { in } \\
0 \\
1\end{array}$ \\
\hline 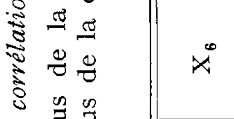 & $\begin{array}{l}\mathscr{o} \\
\stackrel{0}{0}\end{array}$ & $\stackrel{2}{\circ}$ & $\stackrel{8}{\stackrel{8}{\circ}}$ & 点 & $\begin{array}{l}8 \\
0 \\
0 \\
0\end{array}$ & & $\begin{array}{l}\mathscr{E} \\
\dot{0}\end{array}$ & 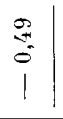 & $\stackrel{8}{8}$ & $\frac{9}{i}$ & $\begin{array}{c}0 \\
8 \\
0 \\
0 \\
1\end{array}$ & $\begin{array}{l}\text { in } \\
\text { in } \\
1\end{array}$ \\
\hline 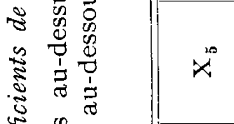 & $\stackrel{m}{\vec{c}}$ & 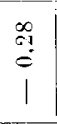 & $\stackrel{2}{0}$ & $\stackrel{+}{=}$ & & $\overrightarrow{5}$ & $\begin{array}{c}+ \\
\infty \\
0 \\
0\end{array}$ & $\stackrel{s}{8}$ & 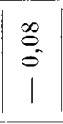 & $\vec{E}$ & $b_{0}$ & $\stackrel{ \pm}{5}$ \\
\hline 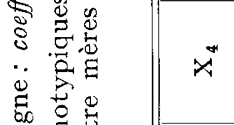 & E & $\stackrel{\Xi}{\circ}$ & $\begin{array}{l}D \\
0 \\
0\end{array}$ & & $\stackrel{̃}{*}$ & $\stackrel{\substack{0 \\
\text { an } \\
0}}{1}$ & 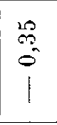 & $\stackrel{?}{\stackrel{0}{0}}$ & $\stackrel{8}{\circ}$ & $\begin{array}{l}\stackrel{20}{9} \\
\stackrel{9}{=} \\
1\end{array}$ & $\mid$ & 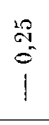 \\
\hline 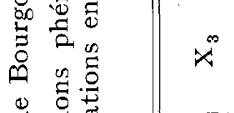 & $\begin{array}{l}0 \\
2 \\
0 \\
0\end{array}$ & $\begin{array}{l}\stackrel{0}{1} \\
\stackrel{1}{0}\end{array}$ & & $\begin{array}{l}\infty \\
\infty \\
0 \\
0\end{array}$ & $\frac{m}{0}$ & 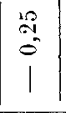 & $\frac{2}{0}$ & $\frac{2}{2}$ & $\stackrel{n}{\stackrel{n}{0}}$ & 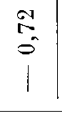 & $\stackrel{10}{0}$ & $\overbrace{1}^{20}$ \\
\hline 焉弯 & $\begin{array}{l}\infty \\
\infty \\
\infty \\
0\end{array}$ & & $\stackrel{\sigma_{0}^{+}}{\sigma_{0}}$ & $\begin{array}{l}\infty \\
\infty \\
0 \\
0\end{array}$ & $\stackrel{8}{i}$ & $\mid \begin{array}{l}12 \\
0 \\
0 \\
1\end{array}$ & $\begin{array}{l}5 \\
0 \\
0 \\
1\end{array}$ & 告 & 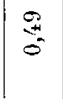 & $\stackrel{E}{E}$ & $\mid \begin{array}{l}20 \\
2 \\
0 \\
1\end{array}$ & 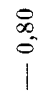 \\
\hline$\vec{x}$ & & $\stackrel{5}{\delta}$ & $\stackrel{s}{\delta}$ & $\frac{\infty}{0}$ & $\frac{a}{\sigma}$ & $\stackrel{5}{0}$ & $\stackrel{\theta}{0}$ & $\begin{array}{l}\stackrel{8}{i g} \\
0 \\
0\end{array}$ & $\stackrel{20}{+}$ & $\begin{array}{l}20 \\
0 \\
0\end{array}$ & $\begin{array}{l}8 \\
0 \\
0 \\
1\end{array}$ & $\begin{array}{l}8 \\
0 \\
0 \\
0\end{array}$ \\
\hline & $\vec{x}$ & $x^{11}$ & $x^{\infty}$ & $\vec{x}$ & $x^{0}$ & $x^{i}$ & $\tilde{x}$ & $x^{\infty}$ & $\ddot{x}$ & $\underset{x}{x}$ & $\vec{x}$ & $x^{5}$ \\
\hline 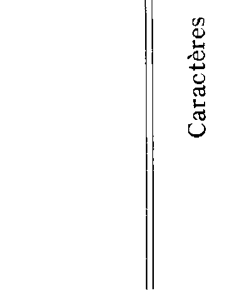 & 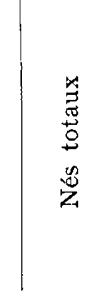 & 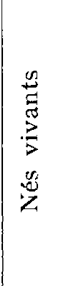 & $\begin{array}{l}\bar{a} \\
z\end{array}$ & $\begin{array}{l}\overline{6} \\
i s \\
z\end{array}$ & $\underbrace{\dot{\circ}}$ & 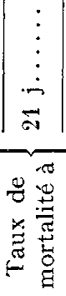 & $\dot{\dot{0}}$ & $\mid \begin{array}{c}\dot{\vec{s}} \\
\frac{y}{0} \\
\dot{0}\end{array}$ & $\underbrace{\frac{\dot{0}}{0}}_{\infty}$ & 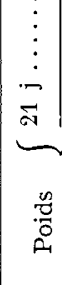 & 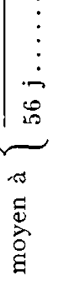 & 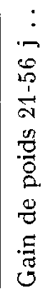 \\
\hline
\end{tabular}


En race Néo-Zélandaise, la taille de portée à la naissance et le nombre de nés vivants par portée sont fortement liés aux taux de mortalité à 2 I et 56 jours $(r=0,53$ à 0,87$)$ mais linéairement indépendants du nombre de lapereaux dans les portées à ces âges-là. Nous avons une situation inverse en race Fauve de Bourgogne, les tailles de portées à la naissance (nés totaux, nés vivants par portée) étant en corrélations faibles ou nulles avec les taux de mortalité à $2 \mathrm{I}$ et 56 jours, et en corrélations positives très élevées avec les tailles de portée à $2 \mathrm{I}$ et 56 jours.

Le gain pondéral moyen par portée d'une descendance de mère est indépendante des effectifs par portée (très faiblement lié à 56 jours) en race Néo-Zélandaise blanche. Par contre, en race Fauve de Bourgogne, il est d'autant plus faible que les tailles de portée sont plus élevées, l'intensité de la liaison diminuant avec l'âge des lapereaux $(r=0,80$ avec le nombre de nés vivants par portée et $r=-0,25$ avec la taille de la portée à 56 jours).

\section{DISCUSSION}

\section{I. - Signification zootechnique des caractères mesurés}

Les nombres de lapereaux nés totaux et nés vivants par portée permettent d'exprimer la prolificité des lapines, et la mortalité à la naissance. Les lapereaux considérés comme morts à la naissance sont ceux trouvés morts lors de la première visite du nid par l'éleveur, qui a lieu en général dans les I 2 à 24 heures qui suivent la mise bas. Il n'a pas été possible, dans cette étude, de séparer, parmi les morts à la naissance, ceux qui sont morts avant, pendant ou après la parturition. Cette distinction sera utile à faire au niveau d'un élevage expérimental. Le taux de mort-nés (7,40 p. IOo) observé ici, sur les 5942 portées, correspond à celui $(8,85$ p. IOO) indiqué par D. B. Sir'rimann et al. (Ig64) et obtenu à la station expérimentale de Fontana en Californie. Des études plus fines ultérieurement devront préciser si ce taux est susceptible de varier suivant les conditions d'élevage, la prolificité, la race ou la famille génétique.

Le poids total de la portée à l'âge de $2 \mathrm{I}$ jours des lapereaux est une mesure indirecte de la production laitière de la lapine, comme l'ont confirmé les résultats de LEBAS (I969). Sur le plan génétique, la mesure de l'aptitude laitière des mères devra aussi tenir compte de l'effectif de lapereaux dans la portée à l'âge de 2r jours, 1e poids total de la portée lui étant évidemment fortement lié. Cependant la corrélation $(r=0,59$ ou 0,73$)$ n'est pas assez forte pour que l'effectif de portée seul puisse remplacer son poids total à l'âge de 2 I jours. Compte tenu de l'évolution actuelle vers un sevrage des lapereaux dès l'âge de 28 jours, la pesée de la portée à 1'âge de 2 I jours pourrait être remplacée par une pesée à 28 jours, ce qui présenterait des avantages pratiques (une seule manipulation pour les opérations d'identification, pesée, sevrage). Le poids de portée à 28 jours est également en corrélation élevée avec la production laitière. Le poids moyen individuel par portée, à $2 \mathrm{I}$ ou 28 jours peut être interessant à considérer comme caractère à sélectionner, les animaux ne devant pas être trop légers au sevrage.

Enfin les lapereaux sont pesés individuellement à 56 jours pour obtenir une 
information sur la vitesse de croissance individuelle dans le jeune âge. Nous avons cependant analysé ici ce caractère en tant que caractère maternel, bien que le sevrage s'effectue dans la plupart des cas avant 56 jours, en considérant le poids total et l'effectif de portée à 56 jours. La pesée à 56 jours est pratiquée aux États-Unis, compte tenu d'une production de lapins à frire. Elle serait avantageusement remplacée, en France, par une pesée à 70 jours ou 77 jours, les lapereaux destinés à la boucherie n'étant pas commercialisés avant cet âge, dans nos conditions.

\section{2. - Validité du modèle interprétatif.}

Forme des distributions de fréquence

Nous avons, par approximations et calculs statistiques successifs, cherché le modèle suivant lequel l'analyse des données nous permet d'admettre l'hypothèse de l'inexistence d'interactions. Cela nous a permis d'estimer les valeurs des effets fixés mois-année de mise bas et numéro de portée, et d'utiliser ces estimations pour " corriger " les données. En effet, dans un élevage et pour une race donnée, en moyenne, l'influence du numéro de portée est indépendante de la date de naissance des lapereaux. Cependant, ces résultats doivent être considérés avec prudence. En effet, les tests de signification des effets ne sont strictement valables que sous l'hypothèse de

Valeurs des coefficients

\begin{tabular}{|c|c|c|c|c|c|c|c|c|c|c|c|}
\hline \multicolumn{3}{|c|}{ Elevage $n^{\circ}$} & \multicolumn{3}{|c|}{1} & \multicolumn{3}{|c|}{2} & \multicolumn{3}{|c|}{3} \\
\hline \multicolumn{3}{|c|}{ Caractères } & $N$ & $\sqrt{b_{1}}$ & $b_{2}$ & $\mathrm{~N}$ & $\sqrt{b_{1}}$ & $b_{2}$ & $N$ & $\sqrt{b_{1}}$ & \\
\hline Nés & totaux & $\mathrm{X}_{1}$ & 1335 & $-0,27 *$ & 2,91 & 589 & $-0,20$ & 3,25 & 521 & $-0,11 *$ & 2,7 \\
\hline Nés & ivants & $\mathrm{X}_{2}$ & 1335 & $-0,10^{*}$ & 2,94 & - & $-0,52 *$ & 3,34 & - & $-0,25 *$ & 2,6 \\
\hline & $21 \mathrm{j}$ & $X_{3}$ & 1335 & $-0,51 *$ & $2,73^{*}$ & - & $-0,50 *$ & 2,77 & - & $-0,00$ & ,2 \\
\hline & $56 \mathrm{j}$ & $X_{4}$ & 1335 & $-0,42 *$ & $2,61 *$ & - & $-0,48 *$ & 2,75 & - & $-0,08$ & 2,2 \\
\hline \multirow{3}{*}{$\begin{array}{l}\text { Taux de } \\
\text { mortalité à }\end{array}$} & $0 \mathrm{j} \ldots$. & $\mathrm{X}_{5}$ & 1335 & $3,05 *$ & $13,7 *$ & - & $2,66 *$ & $12,8 \quad *$ & - & $1,32 *$ & 4,0 \\
\hline & $21 \mathrm{j}$. & $\mathrm{X}_{6}$ & 1335 & $1,05 *$ & $3,62 *$ & - & $1,19^{*}$ & $4,67 *$ & - & $0,24^{*}$ & 2,1 \\
\hline & $56 \mathrm{j}$. & $X_{7}$ & 1335 & $0,74 *$ & 2,99 & - & $1,03 *$ & $4,18^{*}$ & - & $0,0^{\prime}$ & \\
\hline \multirow{2}{*}{$\begin{array}{l}\text { Poids } \\
\text { totaux à }\end{array}$} & $21 \mathrm{j} .$. & $X_{8}$ & 1199 & $-0,67 *$ & $3,71 *$ & 531 & $-0,69 *$ & $3,78 *$ & 375 & $-0,53 *$ & 3,4 \\
\hline & $56 \mathrm{j} \ldots$ & $X_{9}$ & 1176 & $-0,82 *$ & 2,82 & 525 & $-0,49^{*}$ & 3,06 & 367 & 0,04 & 2,8 \\
\hline \multirow{2}{*}{$\begin{array}{l}\text { Poids } \\
\text { moyen à }\end{array}$} & $21 \mathrm{j} \ldots$ & $X_{10}$ & 1199 & $0,87^{*}$ & $4,29 *$ & 531 & $0,72 *$ & $5,21 *$ & 375 & $0,5_{4}^{\prime} *$ & 3,8 \\
\hline & $56 \mathrm{j} \ldots \ldots$ & $\mathrm{X}_{11}$ & 1176 & $-0,01 *$ & $3,52 *$ & 525 & $0,19 *$ & $3,59 *$ & 367 & $0,33 *$ & 3,1 \\
\hline \multicolumn{2}{|c|}{ Gain de poids $21-56 \mathrm{j} \ldots$} & $\mathrm{X}_{12}$ & 1176 & $-0,42 *$ & $3,79 *$ & - & $0,04_{4}^{4}$ & $3,99 *$ & - & 0,15 & \\
\hline
\end{tabular}

* Valeur significativement différente de la valeur théorique dans le cas de la loi normale. 
normalité des distributions des écarts au modèle (résidus). Nous avons pu étudier la forme de distribution des valeurs brutes des caractères et des variables transformées (Arc sinus $\sqrt{ }^{-}$) pour les pourcentages. Le tableau 8 présente les valeurs des paramètres (coefficients de Pearson) d'asymétrie $\sqrt{b_{1}}$ et d'aplatissement $b_{2}$ (voir par exemple, DAGNELIE, I969) pour chaque caractère et élevage.

$\mathrm{Si} \sqrt{b_{1}}$ est négatif, la distribution présente un déficit d'individus ayant des valeurs supérieures à la moyenne (dissymétrie à gauche), dans le cas contraire le déficit a lieu pour des valeurs inférieures à la moyenne (dissymétrie à droite). Le coefficient d'aplatissement $b_{2}$ prend la valeur 3 pour une loi normale; s'il est supérieur, les classes centrales de la distribution renferment une proportion d'individus supérieure à la normale (distribution trop pointue), dans le cas contraire les classes extrêmes ont des effectifs trop importants par rapport aux classes centrales (distributions aplaties). D'une façon générale, les effectifs de portée aux différents âges sont dissymétriques à gauche et ces distributions ont tendance à s'aplatir lorsque l'âge des lapereaux augmente. Il en est de même pour les poids totaux de portée aux âges de $2 \mathrm{I}$ et 56 jours. Dans la distribution des effectifs par portée, il y a donc plus de valeurs inférieures à la moyenne, que de valeurs supérieures. Le même phénomène observé pour les poids totaux de portée s'explique du fait de la corrélation entre

$\sqrt{b_{1}}$ et d'aplatisseme:nt $b_{2}$

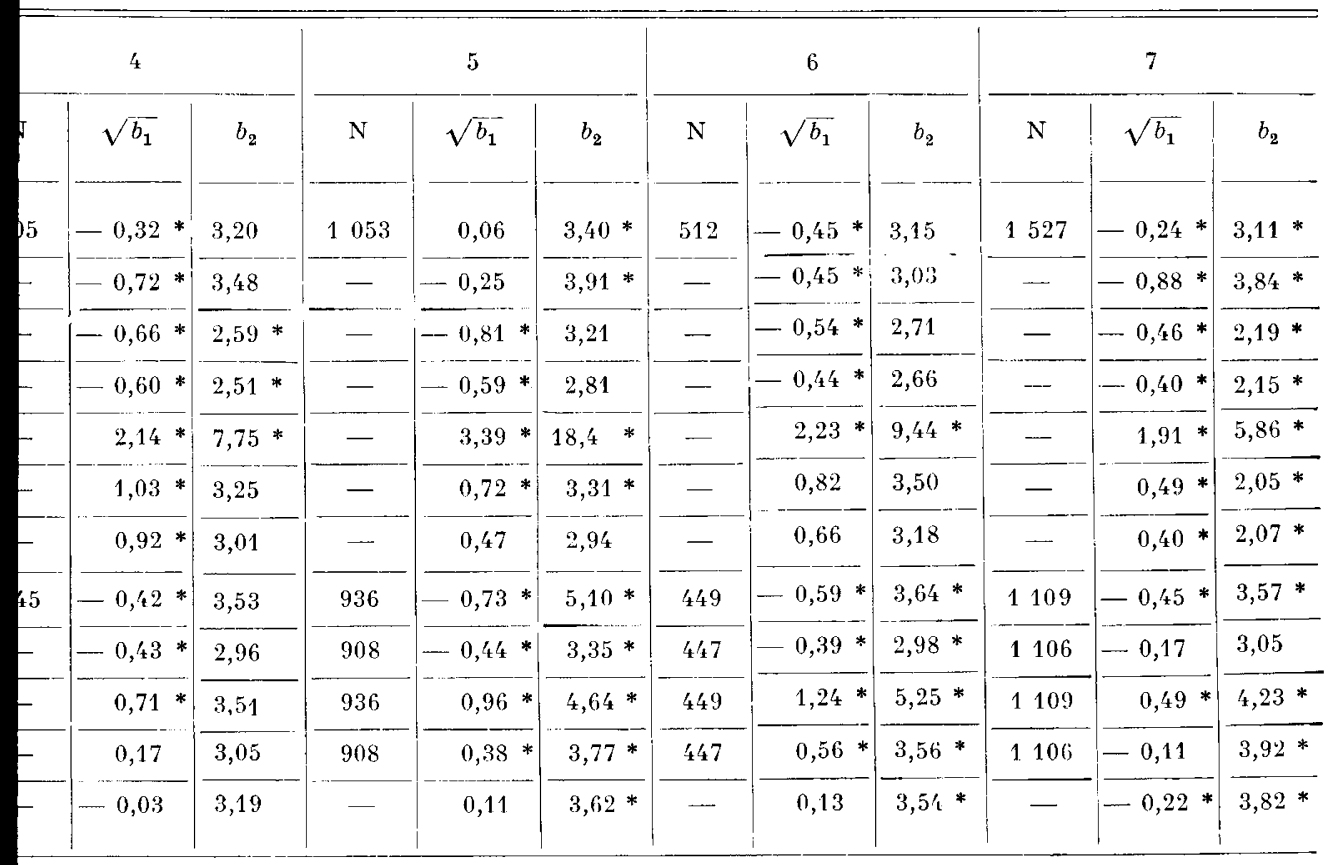


effectif de portée et poids total de portée. Le fait que la distribution des effectifs de lapereaux par portée s'écarte de la normale est à prendre en considération dans le calcul de l'espérance du progrès génétique à la suite de la sélection. Dans le cas de distributions dissymétriques à gauche (cas des effectifs de lapereaux par portée aux différents âges) et de plus trop "pointues " (cas du nombre de lapereaux nés vivants par portée dans certains cas), l'espérance du progrès génétique sera différente, pour des mêmes valeurs de l'héritabilité et du pourcentage d'animaux sélectionnés, que dans le cas d'une distribution normale de même, pour les distributions plus aplaties que la normale.

\section{3. - Influence des effets du milieu (mois-année de naissance) et du numéro de portée de la lapine}

La mise en évidence d'effets significatifs de la date de naissance des portées et de l'âge de la mère (exprimé par le numéro de portée) sur les performances des portées, confirme en partie les résultats de Sirtiman et al. (I964). Les résultats de ces auteurs ne concernent que les taux de gestation, les nombres de nés totaux, nés vivants et nés morts par portée. Ils trouvent que les trois premiers caractères décroissent depuis un maximum au printemps jusqu'à un minimum en septembre, dans le troupeau de lapines de la station expérimentale de Fontana, en Californie, pendant les années I943 à I960. Ces auteurs ont en plus mis en évidence un parallélisme entre le taux de mortalité à la naissance calculé par mois, dans les portées où il y avait au moins un survivant, et la courbe des températures maximum. Cette relation n'existe pas lorsqu'on considère les taux de mortalité mensuels à partir des portées où tous les lapereaux sont nés morts. Nos résultats traduisent l'influence de la date de naissance des lapereaux sur l'ensemble des caractères étudiés relatifs aux tailles de portée, poids de portée aux différents âges et taux de mortalité. Cependant l'évolution dans le temps des déviations liées aux niveaux de ce facteur (moisannée de naissance) diffère d'un élevage à l'autre et d'une année à l'autre pour un même élevage. Cela traduit l'existence d'interactions entre ce facteur (mois de naissance) et l'effet de l'élevage ou de 1'année, et l'on ne peut pas dégager de tendance générale dans le temps contrairement aux résultats de SirTmaNn et al. (rg64) qui concernaient un seul élevage. La variabilité des conditions de milieu dans le temps masque probablement des variations saisonnières éventuelles. Les influences saisonnières ont encore été peu étudiées sous l'angle expérimental. La photopériode et la température peuvent avoir une influence. WALTER (x968) analyse la fertilité du mâle d'après le taux d'acceptation : la stimulation de l'activité sexuelle du mâle est maximum quand la photopériode claire est minimum et atteint $8 \mathrm{~h}$ sous notre latitude. Elle diminue lorsque la durée quotidienne d'éclairement dépasse $\mathrm{I}_{3} \mathrm{~h}$ 30-I4 h. Chez la femelle, la stimulation de l'activité sexuelle est obtenue dès que la photopériode claire dépasse I $2 \mathrm{~h}$. Elle est maintenue tant que la photopériode claire crô̂t de I $2 \mathrm{~h}$ à $\mathrm{I} 6 \mathrm{~h}$ avec un maximum d'activité sous $\mathrm{I} 6 \mathrm{~h}$ d'éclairement. L'influence des jours courts est défavorable sur la femelle. Ces résultats, sur le lapin domestique, sont comparables à ceux obtenus par WATSON (I957) sur le lapin sauvage. Par ailleurs, certains auteurs ont trouvé que des températures trop élevées $\left(32^{\circ}\right.$ à $\left.34^{\circ} \mathrm{C}\right)$ causent une réduction de l'activité spermatique. 
D’une façon générale (tabl. 4) le numéro de portée de la lapine modifie significativement les moyennes de l'ensemble des performances analysées, comme l'indiquaient déjà les résultats de RoLisins et al. (I963). En moyenne pour les sept élevages étudiés, les résultats des portées numéros I sont inférieurs de 7 et $9 \mathrm{p}$. Ioo respectivement pour les nombres de nés totaux et nés vivants par portée, de I2 et I4 p. Ioo pour les efféctifs par portée à 2 I et 56 jours, de Io à I 2 p. IOO pour les poids totaux de portée à $2 \mathrm{I}$ et 56 jours, de 3 à $4 \mathrm{p}$. Ioo seulement pour les poids moyens individuels par portée et pour le gain de poids du lapereau moyen de la portée. Les taux de mortalité aux différents âges sont supérieurs de $\mathrm{I} 3$ à $20 \mathrm{p}$. IoO en première portée par rapport à la moyenne. Il y a une grande différence entre résultats des $\mathrm{I}^{\mathrm{er}}$ et $2^{\mathrm{e}}$ portées, celle-ci étant la plus importante pour les taux de mortalité, et la moins importante pour les poids moyens individuels par portée et le caractère de croissance. Il faut noter l'incidence du taux de mortalité à la naissance dans les portées numéro I, de 2I,3 p. Ioo supérieure à son incidence moyenne. Dans les portées numéro $I$, il y a à la fois moins de lapereaux nés totaux $(0,56$ en moins par rapport à la moyenne générale), et plus de morts à la naissance, le taux de mortalité à la naissance passant à 9 p. Ioo au lieu de 7,4 p. roo en moyenne générale. Pour l'ensemble des caractères, les résultats sont légèrement meilleurs en portée numéro 3 qu'en portée numéro 2 , les productions maximales s'observant en troisième et quatrième portées. Les performances des lapines en cinquième portée sont très variables d'un élevage à un autre et 1 'on ne peut pas en tirer de règle générale. D'ailleurs, à ce stade, les variations de production traduisent de moins en moins le seul effet de l'âge des lapines, à cause de la sélection naturelle ou artificielle qui s'opère. L'amélioration des performances à partir de la portée numéro 2, correspond à une augmentation de la prolificité et de la production laitière des lapines, augmentation classiquement notée chez d'autres espèces. La diminution du taux de morts à la naissance, continuelle jusqu'à la cinquième portée, peut traduire une meilleure préparation physiologique et du comportement (préparation du nid) de la lapine à la mise bas.

\section{4. - Liaisons phénotypiques entre les caractères}

Il n'existe pas dans la littérature de données aussi complètes sur les liaisons entre ces caractères relatifs à la prolificité des lapines, viabilité et croissance des lapereaux jusqu'à l'âge de 56 jours. I1 faut noter l'intérêt d'obtenir des effectifs de nés vivants par portée élevés (Io à 12 ). Bien que le taux de mortalité soit en moyenne un peu plus élevé (la corrélation est très faible) dans les portées où il y a plus de lapereaux vivants à la naissance, ces portées sont aussi celles où il y a le plus de lapereaux sevrés et présents à 56 jours. Par ailleurs la vitesse de croissance de 1'animal moyen de la portée, entre $2 \mathrm{I}$ et 56 jours, n'est pas, ou peu ralentie dans ces portées. Une mortalité pré-sevrage plus forte dans les portées de fort effectif peut être due au fait que les poids des lapereaux dans ces portées sont plus faibles. LEBAS (I97I) a en effet montré que la probabilité de survie des lapereaux diminuait lorsque leur poids à la naissance diminuait. Il faut cependant noter que les corrélations avec les taux de mortalité sont à interpréter avec circonspection. Celles-ci ne traduisent qu'une tendance linéaire moyenne non vérifiée dans les extrémités des distributions et l'incidence de la prolificité sur la viabilité des lapereaux devra être étudiée plus en détail. 
La corrélation élevée entre les nombres de lapereaux vivants par portée aux âges de $2 \mathrm{I}$ et 56 jours, nettement plus élevée qu'entre la naissance et $2 \mathrm{I}$ jours, traduit le fait que la taille de portée de chaque lapine varie peu après 1'âge de 2 I jours. Cela rejoint les résultats de LEBAS (I97I) qui indique que la plus grande partie de la mortalité des jeunes intervient lors de leur première et deuxième semaine de vie. La prolificité des lapines n'est donc pas le seul critère qui conditionne la taille de portée à 2 I et 56 jours. Les tailles de portée à ces deux âges dépendent aussi des taux de mortalité des lapereaux et donc de leur viabilité. Ce deuxième caractère apparaissant au moins aussi important que la prolificité.

\section{5. - Corrélations dues aux effets des mères}

Une plus grande prolificité des mères conduit à des poids totaux de portée plus élevée aux âges de 21 et 56 jours, ces poids totaux de portée étant évidemment en corrélation positive avec la taille de portée à ces âges. Cela indique que dans la comparaison des lapines sur leur aptitude laitière d'après le poids total de portée à 2I jours, il faut tenir compte du numéro de portée des lapines comparées, mais aussi de la taille de portée à cet âge. Par contre, les poids moyens individuels par portée des lapereaux aux âges de 21 et 56 jours, sont en corrélation négative avec les effectifs à ces âges. Une taille de portée plus élevée s'accompagne donc d'un poids individuel des lapereaux plus faible, en moyenne, notamment au sevrage, lorsque celui-ci a lieu à âge fixe. Dans le cas d'un sevrage relativement précoce (par exemple à l'âge de 28 jours), le poids individuel des lapereaux ne doit pas être trop faible. Dans les programmes de sélection, il semble donc souhaitable de chercher à augmenter simultanément la taille de portée et le poids moyen individuel des lapereaux par portée au sevrage, ces deux caractères variant, phénotypiquement, en sens inverse.

Les corrélations dues aux effets des mères diffèrent parfois des corrélations phénotypiques, aussi est-il utile de les discuter. Les relations entre critères de croissance sont différentes d'une race à l'autre. En race Néo-Zélandxise il existe entre les caractères de croissance pris deux à deux, des liaisons linéaires positives. Les lapines ayant un poids total de portée à $2 \mathrm{I}$ jours élevé, sont mères de lapereaux à poids moyen plutôt supérieurs à 2 I jours $(r=0,32)$, supérieurs à 56 jours $(r=0,75)$, dans une portée lourde $(r=0,75)$, et qui croîtront rapidement $(r=0,94)$. Par contre, pour les lapines Fauve de Bourgogne de cet élevage, le poids total de la portée à $2 \mathrm{I}$ jours n'indique rien sur les poids moyens des lapereaux à $2 \mathrm{I}$ jours $(r=-0, \mathrm{I} 9)$ et à 56 jours $(r=0,09)$, mais le poids total de la portée se rangera à 56 jours de la même manière qu'à 2 I jours $(r=0,97)$, la liaison avec le gain de poids du lapereau moyen étant faible $(r=0,2 \mathrm{I})$. Le gain de poids du lapereau moyen d'une portée est en liaison négative avec la taille de portée en race Fauve de Bourgogne de cet élevage, alors que ce n'est pas le cas en race Néo-Zélandaise blanche. Ces résultats indiquent que si les facteurs génétiques et maternels agissent dans le même sens sur le poids total de la portée et la croissance des lapereaux de ces portées, par contre, dans l'élevage Fauve de Bourgogne, la production laitière insuffisante des mères serait un facteur limitant la croissance individuelle des lapereaux, qui ne pourraient extérioriser leurs aptitudes de croissance, lorsqu'ils sont sous la mère.

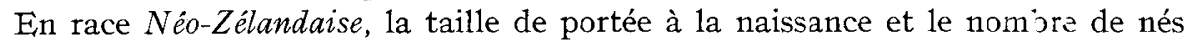
vivants sont fortement liés, positivement, au taux de mortalité à 56 jours $(r=0,72$; 
$0,87)$ mais indépendants de l'effectif de lapereaux présents dans chaque portée à cet âge $(r=0,04 ;-0,06)$; en Fauve de Bourgogne, les deux premiers critères sont pratiquement non corrélés, les deux suivants sont fortement liés $(r=0,78 ; 0,88)$. Or les variances de la taille de portée à la naissance, sont de 5,64 pour les Néo-Zélandais blancs et de 3,47 pour les Fauves de Bourgogne; celles de la taille de portée à 56 jours sont respectivement pour les deux races de 4,70 et 3,77. Tout se passe comme si dans les descendances de femelles Néo-Zélandaises il y avait une tendance à une homogénéisation des tailles de portée à la naissance, cette liaison n'existant pas en Fauve de Bourgogne. Cela s'accompagne, dans cet élevage de Fawve de Bourgogne d'une plus grande homogénéité des tailles de portée après la naissance. La relation observée en race Néo-Zélandaise blanche traduit donc une tendance à une homogénéisation des tailles de portée, homogénéisation qui n'existe pas à la naissance, dans cette race, du moins en comparaison avec l'élevage Fauve de Bourgogne. Dans les deux races, les corrélations dues à la mère, et donc aux effets maternels et génétiques, indiquent que les poids plus élevés à 2 I ou 56 jours, ou le gain de poids dans la période $2 \mathrm{I}-56$ jours, sont liés négativement aux taux de mortalité à ces âges. Les portées les plus viables après la naissance sont donc en même temps celles où il y a la meilleure croissance des lapereaux. Ce résultat est logique. Il reste cependant à faire la part, dans la meilleure viabilité, entre ce qui est dû à la vitalité des lapereaux et à 1 'aptitude laitière des mères.

\section{6. - Infuences maternelles et génétiques}

\section{I. Répétabilités dues à la mère.}

La répétabilité est, comme on le sait, une limite supérieure de l'héritabilité. Les valeurs trouvées pour les répétabilités entre mères sont faibles, mais cependant significatives. Pour les caractères relatifs aux aptitudes maternelles et à la viabilité des lapereaux, cela laisse supposer que les héritabilités sont faibles. Cela est en accord avec les résultats de Rolitiss et al. (1963) qui ont trouvé des variances génétiques additives très faibles pour les nombres de nés vivants et sevrés par portée, ainsi que pour le poids total de portée au sevrage. Cette étude concernait I 472 portées de lapines de race Néo-Zélandaise blanche, le sevrage des lapereaux intervenait à l'âge de 56 jours. En ce qui concerne nos données, la répétabilité est plus élevée pour le nombre de lapereaux nés totaux que pour les autres effectifs de lapereaux par portée, et notamment les nés vivants. On peut penser que le nombre de nés totaux est connu avec plus d'exactitude que celui des nés vivants, le dénombrement des lapereaux vivants dans la portée intervenant à des temps variables après la mise-bas. Le caractère nombre de nés totaux est donc intéressant à considérer, tant que l'on ne peut pas connaître avec précision la mortalité périnatale.

Les composantes mère des répétabilités des taux de mortalité sont significativement supérieures à 0 , quoique faibles. Il y a donc un effet significatif de la mère sur la viabilité de ses lapereaux au cours de ses portées successives. Cela peut être en relation avec des effets génétiques traduisant une meilleure résistance aux maladies de certains génotypes. Rolins et CASADY (I967) ont trouvé une composante père de l'héritabilité égale à $0,12 \pm 0,02$ pour la mortalité des lapereaux à cause d'entérite ou de pneumonie entre 15 et 56 jours d'âge. La composante mère est plus élevée et 
égale à $0,5^{8} \pm 0,05$. La différence entre les deux composantes traduit la présence d'effets génétiques autres d'additifs, ou d'effets maternels. Dans le cas présent, nous n'avons pas calculé les valeurs des héritabilités. Ce travail, qui ferait appel aux techniques statistiques développées, par exemple par ROBERTSON et LERNER (I949) ou par Bogyo et BECKER (I965) devrait être effectué, dans un premier temps, sur les données d'un élevage expérimental où les conditions de mortalité ainsi que les lésions corporelles observées sur les animaux pourraient être mieux précisées. Les répétabilités des poids totaux de portée et du lapereau moyen des portées, sont du même ordre de grandeur que celles relatives aux effectifs par portée. Cela s'explique du fait des corrélations élevées entre les effectifs par portée et ces poids, surtout entre effectifs et poids totaux de portée.

De même pour le gain de poids du lapereau moyen de la portée entre 2 I et 56 jours la répétabilité est du même ordre de grandeur.

Il faut noter que les valeurs des répétabilités sont plus élevées, dans notre échantillon, dans l'élevage Fauve de Bourgogne qu'en Néo-Zélandais; cela indique une plus grande possibilité de progrès génétique en Fauve de Bourgogne qu'en Néo-Zélandais pour les élevages considérés, et sous réserve que les hérétabilités se classent de la même façon que les répétabilités.

\section{2. Effet direct du père.}

Il faut signaler l'existence d'un effet direct du père sur le nombre de lapereaux nés et surtout nés vivants dans l'élevage Fauve de Bourgogne, qui mériterait d'être étudié plus à fond. Il peut être dû soit à une meilleure fécondance du sperme que l'on pourrait appréhender par l'étude de la concentration en spermatozoïdes, de leur motilité, du pourcentage d'anormaux ; soit à un effet du père sur la mortalité embryonnaire, ce qui n'est pas invraisemblable puisqu'il y a aussi un effet sur le taux de mortalité des lapereaux à la naissance ; soit puisque l'ovulation est provoquée chez cette espèce, à un effet du mâle lié à son comportement; soit à l'ensemble de ces phénomènes agissant simultanément. L'existence d'un effet direct du mâle sur la prolificité des femelles auxquelles il est accouplé a déjà été signalé par LEGAULT et OlLIVIER (I965) chez les porcins. Cet effet direct du père, sur la prolificité des lapines auxquelles il est accouplé, ou sur la viabilité de ses descendants, est intéressant à considérer même s'il est faible. Il conviendra de rechercher si son existence se confirme, dans d'autres échantillons.

L'effet direct du père sur les variables poids et croissance considérées est faible, et en particulier plus faible que celui de la mère. On ne pourrait estimer des héritabilités à partir des valeurs des composantes père de la variance pour ces caractères que si les tailles de portée étaient constantes pour toutes les lapines. Les poids de portée, du lapereau moyen de la portée, doivent donc être comparés après correction pour l'effet taille de portée, donc à taille de portée constante. Ainsi LEPLÈGE (Ig69) a établi la méthode pour le calcul d'un index de sélection sur le caractère vitesse de croissance des descendants dans la période $2 \mathrm{I}-56$ jours, pour des portées ramenées à effectif constant. Cependant nous n'avons pas retrouvé les valeurs élevées des composantes pères de 1'héritabilité des caractères poids trouvées par cet auteur. En fait, compte tenu des tailles variables de portée, les héritabilités des caractères poids devraient s'obtenir par analyse des poids individuels des lapereaux plutôt que par analyse des poids du lapereau moyen de chaque portée. 


\section{CONCLUSION}

I1 ressort de cette première analyse statistique des performances d'élevage des lapines de race Néo-Zélandaise blanche et d'un élevage Fauve de Bourgogne, d'après les résultats de contrôle des performances des adhérents du S. N. E. A. L. C., un certain nombre de conclusions :

Disposant des résultats d'un seul élevage Fauve de Bourgogne, nous n'avons pas voulu faire de comparaisons entre les deux races Néo-Zélandaise blanche et Fauve de Bourgogne, comparaisons qui seraient ici erronées.

L'élevage, la date de naissance des lapereaux, le numéro de portée des lapines modifient les performances zootechniques. L'effet du mois de mise bas, pour un élevage donné, n'est pas le même d'une année à l'autre. Ces interactions rendraient difficile l'utilisation de facteurs de correction pour ces effets. Un effet numéro de portée et âge de la lapine a été mis en évidence.

L'effet direct du père sur la prolificité des lapines et le taux de morts à la naissance, est intéressant à considérer. Sa mise en évidence, au niveau d'un mâle particulier, nécessiterait soit que celui-ci ait donné un grand nombre de portées, soit que l'on fasse appel à des critères plus sensibles à l'influence directe du père. Les répétabilités dues à la mère permettent d'escompter juger de la valeur d'une femelle sur un petit nombre de portées (les quatre premières portées dans le cadre du S. N. E. A. L. C).

L'analyse a donc permis de préciser l'intérêt des différents caractères mesurés notamment des effectifs de lapereaux nés totaux et nés vivants, à 2 I jours, du poids de portée à $2 \mathrm{I}$ jours. Les effectifs et poids de portée à 56 jours n'apportent guère plus d'information que celle de ces caractères mesurés à $2 \mathrm{I}$ jours. Leur prise en compte a cependant été utile pour définir une vitesse de croissance du très jeune animal, en attendant que la pesée à 56 jours soit remplacée, éventuellement, par une pesée à l'âge commercial plus tardif. Il serait maintenant nécessaire d'estimer les héritabilités des caractères relatifs aux aptitudes maternelles des lapines de façon à pouvoir calculer le progrès génétique attendu suivant diverses méthodes de sélection. Il apparaît déjà que la sélection sur les performances d'élevage des lapines devra se faire dans des troupeaux d'effectifs importants, en utilisant des méthodes de sélection familiale ou combinée (faibles valeurs des héritabilités) et en éliminant le facteur du milieu perturbateur dû à l'effet de la période de naissance des lapereaux, ainsi que l'effet du numéro de portée. Nous avons dans ce but fait une première discussion sur l'optimum du plan d'accouplement (PoujARDIEU et RouviER, I972).

Rę̧u pour publication en novembre 1972.

\section{REMERCIEMENTS}

Nous remercions toutes les personnes qui ont contribué à la réalisation de ce travail, et notamment le S. N. E. A. L. C. et son technicien, qui ont recueilli et vérifié les données. 


\section{SUMMARY}

\section{STATISTICAL ANALYSIS OF THE BREEDING PERFORMANCES OF FEMALE RABBITS :} ENVIRONMENTAL FACTORS, CORRELATIONS, REPETABILITY

The breeding performances of New Zealand white ard Fauve de Bourgogne female rabbits are statistically analyzed using the control performance results gathered by members of the National Union for Breeding and Improvement of Meat Rabbits (5942 litters).

The following characters are studied : total number of young rabbits born and the number born alive per litter; size of litter at $2 \mathrm{I}$ and 56 days; mortality rate of young rabbits at birth and at $2 I$ and 56 days ; total weight of litter at $2 I$ and 56 days ; individual mean weight per litter of young rabbits at $2 \mathrm{I}$ and 56 days ; weight gain of average young rabbit in the litter between $2 \mathrm{I}$ and 56 days. The effects of the following factors (considered as constant) and their interactions were tested : year (4 years) and month of birth, breeding number, number of order of the litter. Because of interactions, we had to base the analysis on the test and estimation of the effects of the month-year of birth and litter number ( $1-5)$ for each separate breeding. The study of frequency distribution of data corrected for the effects of these two factors, for each separate breeding, shows that characters relating especially to prolificacy and litter size deviate significantly from the normal and present left asymmetry. This affects the calculation of hope of genetic progress based on selection.

Hierarchically analyzing variance of the same corrected data, we obtained the variance constituents due to effects of breeding and of the sire and dam of the litters.

The breeding and date of birth of the young rabbits modify animal production performance, these two factors being in interaction. Moreover, for a given breeding, there are interactions between the effect of the year and month of birth. The does give the best results in litters 3 and 4 . The worst results are obtained in primiparae which have, in particular, a higher mortality rate at birth.

Repeatability due to maternal effect, for the different characters, is calculated, as well as correlation. Although low, maternal repeatability is significant, which leads us to suppose low values of heritability for these characters. Maternal effect acts in the same way for the viability and growth of the young rabbits up to 56 days. Direct paternal effect on the number of young rabbits born and those born alive has been demonstrated, as well as the mortality rate of young rabbits at birth.

As regards phenotype, prolificacy and litter size at different ages are in negative correlation with individual average weights of young rabbits per litter $(2 \mathrm{I}, 56$ days). However, the growth rate of the average young rabbit of the litter, in the 2 I-56 day age interval, depends little on prolificacy and litter size. A much greater prolificacy usually accompanies higher litter size and weight of thel etter at 21 and 56 days, optimum prolificacy being a total of IO-I 2 young rabbits Lorn per litter.

The results found show the value of various characters measured during performance control. They also allow planning methods of selecting for maternal aptitude which are applicable to the rabbit, although the values of their genetic parameters still remain to be defined.

\section{RÉFÉRENCES BIBLIOGRAPHIQUES}

Bogyo T. P., Becker W. A., I965. Estimates of heritability from transformed percentage sib data with unequal subclass numbers. Biometrics, 21, roor-1007.

Dagnelie P., Iэ69. Théorie et méthodes statistiques. Applications agronomiques. Vol. I, J. Duculot, Gembloux, 48 .

LeBAS F., I969. Alimentation lactée et croissance pondérale du lapin avant sevrage. Ann. Zootech. 18, $197-208$.

Lebas F., I971. Le lapereau sous la m’re. Revue avicole, $\mathrm{n1}^{\circ}$ 5, 195-203.

Legault C., Ollivier L., 1955. Résultats préliminaires coicernant l'influence du verrat sur la taille de la portée en insémination artificielle. Ann. Zootech., 14, 40I-408. 
Legault C., 1969. Étude statistique et génétique des performances d'élevage des truies de race Large White. I. Effets du troupeau, de la période semestrielle, du numéro de portée et du mois de naissance. Ann. Génót. Sél. anim., 1, 282-298.

LEPLÈGE M., I963. La sélection massale des lapins sur le caractère vitesse de croissance. Bull. Tech. Inf. Ing. Services agric., 245, 983-994.

Poujardiev B., Rouvier R., I972. Optimisation du plan d'accouplement dans la sélection combinée. Ann. Génét. Sél. anim., 3, 509-5 19.

Robertson A., Lerner I. M., I949. The heritability of all or none traits : viability of poultry. Genetics, 34, 395-4II.

Rollins W. C., Casady R. B., Sittman K., Sittman D. B., x963. Genetics variance component analysis of litter size and weaning weight of New Zeland white rabbits. J. Anim. Sci., 22, 654-657.

Rollins W. C., CASADY R. B., I967. An analysis of preweaning deaths in rabbits with special emphasis o. enteritis and pneumonia. II. Genetic sources of variation. Anim. Prod., 9, 93-97.

Sitrmann D. B., Rollins W. C., Sittman K., Casady R. B., 1964. Seasonal variation in reproductiva traits of New Zealand white rabbits. J. Reprod. Fert., 8, 29-37.

WATion J. S., 1957. Raproduction of the wild rabbit (Oryctolagus cuniculus) in Hawke's bay New Zealand. N.Z.J. Sci. Technol., 38, $45 \mathrm{I}$.

WAlter Marie-Rose, I968. Variation photopáriodique et activité sexuelle du lapin domestique. Thèse $3^{\text {e }}$ cycle 43 p., Fac. Sci., Paris. 